TRANSACTIONS OF THE

AMERICAN MATHEMATICAL SOCIETY

Volume 361, Number 3, March 2009, Pages 1279-1316

S 0002-9947(08)04577-7

Article electronically published on October 10, 2008

\title{
TRIVIAL SOURCE BIMODULE RINGS FOR BLOCKS AND $p$-PERMUTATION EQUIVALENCES
}

\author{
MARKUS LINCKELMANN
}

\begin{abstract}
We associate with any $p$-block of a finite group a Grothendieck ring of certain $p$-permutation bimodules. We extend the notion of $p$-permutation equivalences introduced by Boltje and Xu (2006) to source algebras of $p$-blocks of finite groups. We show that a $p$-permutation equivalence between two source algebras $A, B$ of blocks with a common defect group and same local structure induces an isotypy.
\end{abstract}

\section{INTRODUCTION}

Let $p$ be a prime, and let $\mathcal{O}$ be a complete discrete valuation ring having a quotient field $K$ of characteristic zero and an algebraically closed residue field $k$ of prime characteristic $p$. For any finite $p$-group $P$ and any interior $P$-algebra $A$ we denote by $\mathcal{T}(A)$ the Grothendieck group, with respect to split exact sequences, of finite direct sums of summands of the $A$ - $A$-bimodules $A \underset{\mathcal{O} Q}{\otimes} A$, with $Q$ running over the subgroups of $P$. If $A$ is a source algebra of a block of a finite group algebra $\mathcal{O} G$, then the group $\mathcal{T}(A)$ has a ring structure:

Theorem 1.1. Let $A$ be a source algebra of a block of a finite group with defect group $P$. The tensorproduct over $A$ induces an associative bilinear multiplication on $\mathcal{T}(A)$ through which $\mathcal{T}(A)$ becomes a unitary ring having an augmentation and an antipode.

We will restate and prove the various parts of Theorem 1.1 in greater generality in Corollary 8.2 and Propositions 8.6 and 12.3. We show in Corollary 8.4 that the ring $\mathcal{T}(A)$ does not depend on the choice of a source algebra of the considered block $b \in Z(\mathcal{O} G)$ of a finite group $G$, and is hence an invariant of the block $b$. One may think of $\mathcal{T}(A)$ as a bimodule version for source algebras of the trivial source ring of a finite group and call $\mathcal{T}(A)$ the trivial source bimodule ring of the block $b$. If $A=\mathcal{O} P$, then $\mathcal{T}(A)$ is isomorphic to the Burnside ring of $P$ (cf. Proposition 12.1), which is also the trivial source ring in that case. Results of Alperin-Broué [1] and Broué-Puig [9] imply that a source algebra $A$ of a block with defect group $P$ determines a fusion system on $P$. For $P$ a finite $p$-group, $A$ an interior $P$-algebra and $Q$ a subgroup of $P$, the Brauer construction with respect to the conjugation

Received by the editors December 14, 2006.

2000 Mathematics Subject Classification. Primary 20C20.

(C)2008 American Mathematical Society Reverts to public domain 28 years from publication 
action of $Q$ on $A$ yields an interior $C_{P}(Q)$-algebra $A(\Delta Q)$. In certain cases, this is compatible with the ring structure on $\mathcal{T}(A)$ :

Theorem 1.2. Let $A$ be a source algebra of a block with defect group $P$ and fusion system $\mathcal{F}$. For any fully $\mathcal{F}$-centralised subgroup $Q$ of $P$ the tensorproduct over $A(\Delta Q)$ induces an associative unitary ring structure on $\mathcal{T}(A(\Delta Q))$ and the Brauer construction induces a unitary ring homomorphism $\mathcal{T}(A) \longrightarrow \mathcal{T}(A(\Delta Q))$.

This is proved in Theorems 9.1 and 9.2. The difficult part is to show that the Brauer construction does actually induce a map from $\mathcal{T}(A)$ to $\mathcal{T}(A(\Delta Q))$; the fact that this map is multiplicative then follows from a result in [15. This theorem does not hold, in general, for the block algebra $\mathcal{O} G b$ instead of $A$. For $A, B$ two source algebras of blocks of finite groups having a common defect group $P$, we denote more generally by $\mathcal{T}(A, B)$ the Grothendieck group, with respect to split exact sequences, of finite direct sums of summands of the $A$ - $B$-bimodules $A \otimes B$, with $Q$ running over the subgroups of $P$. Since $A, B$ are symmetric $\mathcal{O Q}$ $\mathcal{O}$-algebras, the $\mathcal{O}$-dual of an $A$ - $B$-bimodule of the form $A \underset{\mathcal{O} Q}{\otimes} B$ is isomorphic to the $B$ - $A$-module $B \otimes A$. In other words, $\mathcal{O}$-duality induces a group isomorphism $\mathcal{O Q}$

$\mathcal{T}(A, B) \cong \mathcal{T}(B, A)$. For $X$ an element in $\mathcal{T}(A, B)$ we denote by $X^{*}$ its image in $\mathcal{T}(B, A)$ under this isomorphism. The map sending a pair $(M, N)$ consisting of an $A$ - $B$-bimodule $M$ and a $B$ - $A$-bimodule $N$ to the $A$-A-bimodule $M \underset{B}{\otimes} N$ induces a bilinear map $-{ }_{B}-$ from $\mathcal{T}(A, B) \times \mathcal{T}(B, A)$ to the Grothendieck group of $A$ $A$-bimodules, again taken with respect to split exact sequences. The following definition is the source algebra version of the concept of a $p$-permutation equivalence between blocks of finite groups, as introduced by Boltje and $\mathrm{Xu}$ [4].

Definition 1.3. Let $A, B$ be source algebras of blocks of finite groups with a common defect group $P$. A p-permutation equivalence between $A$ and $B$ is an element $X \in \mathcal{T}(A, B)$ such that $X_{B} X^{*}=[A]$ in the Grothendieck group of $A$ - $A$ bimodules and $X^{*} \cdot X=[B]$ in the Grothendieck group of $B$ - $B$-bimodules.

A $p$-permutation equivalence between source algebras $A, B$ induces a $p$-permutation equivalence in the sense of Boltje and $\mathrm{Xu}$ [4. The converse is true for principal blocks, but not for arbitrary blocks in general (cf. Corollary 7.5 and Remark 7.6). What one gains by adopting the slightly more restrictive Definition 1.3 is a generalisation of [4,1.11] to arbitrary blocks:

Theorem 1.4. Let $A, B$ be source algebras of blocks b, $c$ of finite groups $G, H$, respectively, having a common defect group $P$ and the same fusion system on $P$. If there is a p-permutation equivalence between the source algebras $A$ and $B$, then the blocks $b$ and c are isotypic.

This is proved in Theoem 10.1; as in [4, we will show that the isotypy between $b$ and $c$ is obtained by applying the Brauer construction to the $p$-permutation equivalence. Following [15, 1.10] or [17, 1.1] a splendid derived equivalence between source algebras $A, B$ of blocks of finite groups with a common defect group $P$ is a derived equivalence given by a bounded two-sided tilting complex $X$ of $A$ $B$-bimodules whose components in each degree are direct sums of summands of the $A$-B-bimodules $A \otimes B$, where $Q$ runs over the subgroups of $P$. A two-sided 
tilting complex $X$ of this form is then called a splendid tilting complex of $A$ - $B$ bimodules. This is a variation of Rickard's version [25] of a splendid equivalence our definition is again slightly more restrictive in that it is relative to a choice of source idempotents. The motivation then, as here, is to make the notion "splendid" work for arbitrary blocks. If $X$ is a bounded complex of $A$ - $B$-bimodules, we set $[X]=\sum_{i \in \mathbb{Z}}(-1)^{i}\left[X_{i}\right]$, where $\left[X_{i}\right]$ is the isomorphism class of the degree $i$ component of $X$ viewed as an element in the Grothendieck group of $A$ - $B$-bimodules. We obtain the result corresponding to [4, 1.5] in this setting:

Theorem 1.5. Let $A, B$ be source algebras of blocks of finite groups having a common defect group $P$ and let $X$ be a splendid tilting complex of $A$-B-bimodules. The image $[X]$ of $X$ in $\mathcal{T}(A, B)$ is a p-permutation equivalence.

This is an immediate consequence of [14,11.4.2]. Combining the present material with a result of Puig [24] yields the following invariance statement:

Theorem 1.6. Let $A, B$ be source algebras of blocks b, $c$ of finite groups $G, H$, respectively, having a common defect group $P$. Suppose that $\mathcal{O} G b$ and $\mathcal{O} H c$ are Morita equivalent via a bimodule with endo-permutation source. Then $\mathcal{T}(A) \cong$ $\mathcal{T}(B)$.

The structure theory of nilpotent blocks 23 in conjunction with Theorem 1.6 implies:

Corollary 1.7. Let $A$ be a source algebra of a nilpotent block with defect group $P$. Then $\mathcal{T}(A) \cong \mathcal{T}(\mathcal{O} P)$.

Further remarks in $\S 13$ include the observation that the Hochschild cohomology and block cohomology of a block are modules over the trivial source bimodule ring of the block (cf. §13.2), as well as considerations on how far off the trivial source bimodule ring is from being commutative (cf. $\S 13.3$ ).

Convention. All $\mathcal{O}$-algebras are assumed to be finitely generated as $\mathcal{O}$-modules, and all modules are finitely generated left modules, unless stated otherwise. Given two $\mathcal{O}$-algebras $A, B$, we assume that for any $A$-B-bimodule $M$ the left and right $\mathcal{O}$-module structure on $M$ coincide; in other words, $M$ can be viewed as an $A \otimes B^{o p_{-}}$ module. Given finite groups $G, H$, an $\mathcal{O} G$-O $H$-bimodule $M$ can be viewed as an $\mathcal{O}(G \times H)$-module, with $(x, y) \in G \times H$ acting on $M$ by sending $m \in M$ to $x m y^{-1}$. In particular, if $M$ is an indecomposable $\mathcal{O} G-\mathcal{O} H$-bimodule, it makes sense to consider its vertices and sources as an $\mathcal{O}(G \times H)$-module.

\section{Fusion SYSTEMS}

As a consequence of results in [1, 9, any block of a finite group gives rise to a fusion system on any of its defect groups (see Remark 4.4 below). When dealing with fusion systems - a concept introduced by Puig in the early 1990's - we follow the terminology in [18, §2]. Given a fusion system $\mathcal{F}$ on a finite $p$-group $P$, a subgroup $Q$ is called fully $\mathcal{F}$-centralised if $\left|C_{P}(Q)\right| \geq\left|C_{P}(\varphi(Q))\right|$ for any $\varphi \in \operatorname{Hom}_{\mathcal{F}}(Q, P)$. By a result of Puig, any fully $\mathcal{F}$-centralised subgroup $Q$ of $P$ gives rise to a fusion system, denoted by $C_{\mathcal{F}}(Q)$, on $C_{P}(Q)$. See e.g. [19] for a more detailed introduction to fusion systems. In this section we collect some elementary facts on centralisers in fusion systems. 
Proposition 2.1. Let $\mathcal{F}$ be a fusion system on a finite p-group $P$, let $Q$ be a fully $\mathcal{F}$-centralised subgroup of $P$ and let $S$ be a subgroup of $C_{P}(Q)$. Then $S$ is fully $C_{\mathcal{F}}(Q)$-centralised if and only if $Q S$ is fully $\mathcal{F}$-centralised.

Proof. Suppose that $S$ is fully $C_{\mathcal{F}}(Q)$-centralised. Let $\psi: Q S \rightarrow P$ be a morphism in $\mathcal{F}$. Denote by $\tau: \psi(Q) \rightarrow Q$ the inverse of the isomorphism $\left.\psi\right|_{Q}:$ $Q \cong \psi(Q)$ induced by $\psi$. Since $Q$ is fully $\mathcal{F}$-centralised, $\tau$ extends to a morphism $\sigma: \psi(Q) C_{P}(\psi(Q)) \rightarrow Q C_{P}(Q)$ in $\mathcal{F}$ (cf. [18]). Note that $\psi(S) \subseteq C_{P}(\psi(Q))$. By construction, we have $\left.\sigma \circ \psi\right|_{Q}=\operatorname{Id}_{Q}$. Thus $\left.\sigma \circ \psi\right|_{S}$ is a morphism in $C_{\mathcal{F}}(Q)$. Since $S$ is fully $C_{\mathcal{F}}(Q)$-centralised, we have

$$
\left|C_{P}(Q S)\right|=\left|C_{C_{P}(Q)}(S)\right| \geq\left|C_{C_{P}(Q)}(\sigma(\psi(S)))\right|=\left|C_{P}(Q \sigma(\psi(S)))\right| .
$$

Now $\sigma$ sends $C_{P}(\psi(Q S))$ to $C_{P}(Q \sigma(\psi(S)))$, and hence

$$
\left|C_{P}(Q \sigma(\psi(S)))\right| \geq\left|C_{P}(\psi(Q S))\right|
$$

which proves that $Q S$ is fully $\mathcal{F}$-centralised. Conversely, suppose that $Q S$ is fully $\mathcal{F}$-centralised. Let $\tau: S \rightarrow C_{P}(Q)$ be a morphism in $C_{\mathcal{F}}(Q)$. That is, $\tau$ extends to a morphism $\sigma: Q S \rightarrow P$ in $\mathcal{F}$ such that $\left.\sigma\right|_{Q}=\operatorname{Id}_{Q}$. Since $Q S$ is fully $\mathcal{F}$-centralised, we have

$$
\left|C_{C_{P}(Q)}(S)\right|=\left|C_{P}(Q S)\right| \geq\left|C_{P}(\sigma(Q S))\right|=\left|C_{P}(Q \tau(S))\right|=\left|C_{C_{P}(Q)}(\tau(S))\right|,
$$

which proves that $S$ is fully $C_{\mathcal{F}}(Q)$-centralised.

Let $P, Q$ be finite $p$-groups, let $\mathcal{F}$ be a fusion system on $P$ and let $\mathcal{G}$ be a fusion system on $Q$. By [6], the category on $P \times Q$ generated by $\mathcal{F}$ and $\mathcal{G}$ is a fusion system on $P \times Q$, denoted by $\mathcal{F} \times \mathcal{G}$. In particular, $\mathcal{F}$ determines a fusion system $\mathcal{F} \times \mathcal{F}$ on $P \times P$.

Proposition 2.2. Let $\mathcal{F}$ be a fusion system on a finite p-group $P$, let $Q$ be a subgroup of $P$, let $\tau \in \operatorname{Hom}_{\mathcal{F}}(Q, P)$ and set $\Delta_{\tau} Q=\{(u, \tau(u)) \mid u \in Q\}$. The subgroup $\Delta_{\tau} Q$ of $P \times P$ is fully $\mathcal{F} \times \mathcal{F}$-centralised if and only if $Q$ and $\tau(Q)$ are fully $\mathcal{F}$-centralised.

Proof. Let $\sigma: \Delta_{\tau} Q \rightarrow P$ be a morphism in $\mathcal{F} \times \mathcal{F}$. By the definition of $\mathcal{F} \times \mathcal{F}$, this means there are morphisms $\varphi \in \operatorname{Hom}_{\mathcal{F}}(Q, P)$ and $\psi \in \operatorname{Hom}_{\mathcal{F}}(\tau(Q), P)$ such that $\sigma(u, \tau(u))=(\varphi(u), \psi(\tau(u)))$ for all $u \in Q$. Clearly $C_{P \times P}\left(\Delta_{\tau} Q\right)=C_{P}(Q) \times$ $C_{P}(\tau(Q))$ and $C_{P \times P}\left(\sigma\left(\Delta_{\tau} Q\right)\right)=C_{P}(\varphi(Q)) \times C_{P}(\psi(\tau(Q)))$. Thus the inequality $\left|C_{P \times P}\left(\sigma\left(\Delta_{\tau} Q\right)\right)\right| \leq\left|C_{P \times P}\left(\Delta_{\tau} Q\right)\right|$ holds for all morphisms $\sigma: \Delta_{\tau} Q \rightarrow P \times P$ in $\mathcal{F} \times \mathcal{F}$ if and only if the inequalities $\left|C_{P}(\varphi(Q))\right| \leq\left|C_{P}(Q)\right|$ and $\left|C_{P}(\psi(\tau(Q)))\right| \leq$ $\left|C_{P}(\tau(Q))\right|$ hold for all $\varphi \in \operatorname{Hom}_{\mathcal{F}}(Q, P)$ and all $\psi \in \operatorname{Hom}_{\mathcal{F}}(\tau(Q), P)$. The result follows.

Proposition 2.3. Let $\mathcal{F}$ be a fusion system on a finite p-group $P$ and let $Q$ be a fully $\mathcal{F}$-centralised subgroup of $P$. Then the fusion systems $C_{\mathcal{F} \times \mathcal{F}}(\Delta Q)$ and $C_{\mathcal{F}}(Q) \times C_{\mathcal{F}}(Q)$ on $C_{P \times P}(\Delta Q)=C_{P}(Q) \times C_{P}(Q)$ are equal.

Proof. Since $Q$ is fully $\mathcal{F}$-centralised it follows from Proposition 2.2 that $\Delta Q$ is fully $\mathcal{F} \times \mathcal{F}$-centralised. Thus both $C_{\mathcal{F} \times \mathcal{F}}(\Delta Q)$ and $C_{\mathcal{F}}(Q) \times C_{\mathcal{F}}(Q)$ are fusion systems on $C_{P \times P}(\Delta Q)=C_{P}(Q) \times C_{P}(Q)$. Let $R$ be a subgroup of $C_{P}(Q) \times C_{P}(Q)$. Denote by $R_{1}, R_{2}$ the images of $R$ in $C_{P}(Q)$ under the two projections of the direct product $C_{P}(Q) \times C_{P}(Q)$ onto its first and second factor, respectively. Let $\varphi: R \rightarrow C_{P}(Q) \times C_{P}(Q)$ be a morphism in $C_{\mathcal{F} \times \mathcal{F}}(\Delta Q)$. That is, $\varphi$ extends to a 
morphism $\psi: R \Delta Q \rightarrow P \times P$ in $\mathcal{F} \times \mathcal{F}$ such that $\left.\psi\right|_{\Delta Q}=\operatorname{Id}_{\Delta Q}$. The images in $P$ of $R \Delta Q$ under the two projections of $P \times P$ onto its factors are equal to $R_{1} Q$ and $R_{2} Q$. Thus $\psi$ being a morphism in $\mathcal{F} \times \mathcal{F}$ is equivalent to the existence of morphisms $\psi_{1}: R_{1} Q \rightarrow P$ and $\psi_{2}: R_{2} Q \rightarrow P$ which are the identity on $Q$ and which induce $\psi$. The result follows.

\section{The Generalised Brauer CONStruCtion}

3.1. The Brauer construction has its origins in Brauer's observation that the canonical linear projection $k G \rightarrow k C_{G}(P)$ induces an algebra homomorphism $Z(k G) \rightarrow$ $Z\left(k C_{G}(P)\right)$, for any finite group $G$ and any $p$-subgroup $P$ of $G$. Broué [7] observed that this extends to a surjective algebra homomorphism $(k G)^{\Delta P} \rightarrow k C_{G}(P)$. This in turn has been extended to $G$-algebras in [9], to modules in [11, and generalised further by Boltje and Külshammer in [3]. We briefly recall the definition and some of the basic properties of this construction, following [3, §2]. Let $P$ be a finite $p$-group and let $\theta: P \rightarrow \mathcal{O}^{\times}$be a group homomorphism. For any $\mathcal{O} P$-module $M$ we set

$$
M^{(P, \theta)}=\{m \in M \mid y m=\theta(y) m(\forall y \in P)\} .
$$

For $\theta=1$ (the trivial group homomorphism) this is the usual $\mathcal{O}$-submodule of $P$-fixpoints in $M$; we write $M^{P}$ instead of $M^{(P, 1)}$. For $Q$ a subgroup of $P$ and $\tau=\left.\theta\right|_{Q}: Q \rightarrow \mathcal{O}^{\times}$we denote by $[P / Q]$ a system of representatives in $P$ of the cosets $P / Q$, and for $m \in M^{(Q, \tau)}$ we set $\operatorname{Tr}_{(Q, \tau)}^{(P, \theta)}(m)=\sum_{y \in[P / Q]} \theta(y)^{-1} y m$. This expression does not depend on the choice of $[P / Q]$ and hence defines an $\mathcal{O}$-linear $\operatorname{map} \operatorname{Tr}_{(Q, \tau)}^{(P, \theta)}: M^{(Q, \tau)} \rightarrow M^{(P, \theta)}$. We set $M_{(Q, \tau)}^{(P, \theta)}=\operatorname{Im}\left(\operatorname{Tr}_{(Q, \tau)}^{(P, \theta)}\right)$. If $\theta=1$ we write $\operatorname{Tr}_{Q}^{P}$ and $M_{Q}^{P}$ instead of $\operatorname{Tr}_{(Q, \tau)}^{(P, \theta)}$ and $M_{(Q, \tau)}^{(P, \theta)}$, respectively. The generalised Brauer construction [3, 2.2] is defined by

$$
M(P, \theta)=M^{(P, \theta)} /\left(\sum_{Q} M_{\left(Q,\left.\theta\right|_{Q}\right)}^{(P, \theta)}+J(\mathcal{O}) M^{(P, \theta)}\right),
$$

where $Q$ runs over the set of all proper subgroups of $P$. The Brauer homomorphism is defined to be the canonical surjection

$$
\operatorname{Br}_{(P, \theta)}^{M}: M^{(P, \theta)} \longrightarrow M(P, \theta) .
$$

If no confusion arises we will write $\operatorname{Br}_{(P, \theta)}$ instead of $\operatorname{Br}_{(P, \theta)}^{M}$. If moreover $\theta$ is the trivial homomorphism we write $M(P)$ instead of $M(P, \theta)$ and $\operatorname{Br}_{P}$ instead of $\operatorname{Br}_{(P, \theta)}$. One can play back this construction to the usual Brauer construction as follows: for $\theta: P \rightarrow \mathcal{O}^{\times}$a group homomorphism, denote by $\bar{\theta}$ the $\mathcal{O}$-algebra automorphism of $\mathcal{O} P$ sending $u \in P$ to $u \theta\left(u^{-1}\right)$. For $M$ an $\mathcal{O} P$-module denote by $\bar{\theta} M$ the $\mathcal{O} P$-module obtained from restricting the action of $\mathcal{O} P$ along $\bar{\theta}$; that is, $\bar{\theta} M=M$ as an $\mathcal{O}$-module, and $y \in P$ acts on $m \in M$ as a multiplication by $y \theta(y)^{-1}$. Then $M^{(P, \theta)}=\left({ }_{\bar{\theta}} M\right)^{P}$, and we have

$$
M(P, \theta)=\left({ }_{\bar{\theta}} M\right)(P) .
$$

Note that if $M$ is a permutation $\mathcal{O} P$-module and if $\theta: P \rightarrow \mathcal{O}^{\times}$is a non-trivial group homomorphism, then $M(P, \theta)=\{0\}$. 
3.2. If $G$ is a finite group having $P$ as a subgroup, then $\mathcal{O} G$ is an $\mathcal{O}(P \times P)$-module with $(u, v) \in P \times P$ acting on $a \in \mathcal{O} G$ as $u a v^{-1}$. For $Q$ a subgroup of $P$ we set $\Delta Q=\{(u, u) \mid u \in Q\}$. This is a subgroup of $P \times P$, and hence acts on $\mathcal{O} G$ (this is the conjugation action of $Q$ on $\mathcal{O} G$ ). The corresponding Brauer construction $(\mathcal{O} G)(\Delta Q)$ is well known to be canonically isomorphic to $k C_{G}(Q)$, and under the canonical identification $(\mathcal{O} G)(\Delta Q)=k C_{G}(Q)$ the Brauer homomorphism

$$
\operatorname{Br}_{\Delta Q}:(\mathcal{O} G)^{\Delta Q} \longrightarrow k C_{G}(Q)
$$

is a surjective algebra homomorphism induced by the linear map sending $x \in G$ to its image in $k C_{G}(Q)$ if $x \in C_{G}(Q)$ and to zero otherwise.

3.3. Let $A$ be an interior $P$-algebra; that is, $A$ is an $\mathcal{O}$-algebra endowed with a group homomorphism $\sigma: P \rightarrow A^{\times}$(or equivalently, with a unitary algebra homomorphism $\mathcal{O} P \rightarrow A)$. Then $A$ becomes an $\mathcal{O}(P \times P)$-module with $(u, v) \in P \times P$ acting on $a \in A$ by $\sigma(u) a \sigma\left(v^{-1}\right)$. For $Q$ a subgroup of $P$, the Brauer construction $A(\Delta Q)$ then becomes an interior $C_{P}(Q)$-algebra, with multiplication induced by that in $A^{\Delta Q}$ via the Brauer homomorphism $\mathrm{Br}_{\Delta Q}^{A}$, and with structural homomorphism

$$
C_{P}(Q) \stackrel{\left.\sigma\right|_{C_{P}(Q)}}{\longrightarrow}\left(A^{\times}\right)^{\Delta Q}=\left(A^{\Delta Q}\right)^{\times} \stackrel{\mathrm{Br}_{\Delta Q}}{\longrightarrow} A(\Delta Q)^{\times} .
$$

If $M$ is an $A$-module, then $M$ can be viewed as an $\mathcal{O} P$-module via $\sigma: P \rightarrow$ $A^{\times}$. The $A$-module structure on $M$ then induces an $A(\Delta Q)$-module structure on the corresponding Brauer construction $M(Q)$, for $Q$ a subgroup of $P$. If $A$ and $B$ are two interior $P$-algebras with structural homomorphisms $\sigma: P \rightarrow A^{\times}$ and $\tau: P \rightarrow B^{\times}$, and if $M$ is an $A$-B-bimodule, then $M$ can be viewed as an $\mathcal{O}(P \times P)$-module with $(u, v) \in P \times P$ acting on $m \in M$ as $\sigma(u) m \tau\left(v^{-1}\right)$. For $Q$ a subgroup of $P$, the $A$ - $B$-bimodule structure on $M$ restricts to an $A^{\Delta Q_{-}} B^{\Delta Q_{-}}$ bimodule structure on $M^{\Delta Q}$ which in turn induces an $A(\Delta Q)-B(\Delta Q)$-bimodule structure on $M(\Delta Q)$. In particular, if $G, H$ are two finite groups having $P$ as a common $p$-subgroup and if $M$ is an $\mathcal{O} G-\mathcal{O} H$-bimodule, we regard $M(\Delta Q)$ as a $k C_{G}(Q)-k C_{H}(Q)$-bimodule through the canonical identifications.

The Brauer construction is functorial, sends $p$-permutation modules to $p$-permutation modules, commutes with duality, and in some cases, commutes with tensor products. See [28, $\S 27, \S 11]$ for more details. Calculating the Brauer construction for $p$-permutation modules is particularly easy:

Lemma 3.4 ([28, (27.6)]). Let $P$ be a finite p-group and let $M$ be an $\mathcal{O} P$-module having a $P$-stable $\mathcal{O}$-basis $X$. Then the image in $M(P)$ of the fixpoint set $X^{P}$ under $\mathrm{Br}_{P}^{M}$ is a k-basis of $M(P)$.

Lemma 3.5 (15, 6.1(iii)]). Let $P$ be a finite p-group and let $M$ be an indecomposable $\mathcal{O} P$-OP -bimodule having a $P \times P$-stable $\mathcal{O}$-basis such that $M$ is projective as a left and right $\mathcal{O} P$-module. Then $M(\Delta P)$ is non-zero if and only if $M \cong \mathcal{O} P$ as $\mathcal{O P}-\mathcal{O P}$-bimodule.

3.6. Let $P$ be a finite $p$-group, let $\theta: P \rightarrow \mathcal{O}^{\times}$be a group homomorphism, let $A$ be an interior $P$-algebra with structural homomorphism $\sigma: P \rightarrow A^{\times}$and let $M$ be an $A$-module. Denote by ${ }_{\bar{\theta}} A$ the interior $P$-algebra which is equal to $A$ as 
an $\mathcal{O}$-algebra, with structural homomorphism sending $y \in P$ to $\sigma(y) \theta\left(y^{-1}\right)$. Note that $\sigma(y)$ and $\sigma(y) \theta\left(y^{-1}\right)$ act in the same way on $A$. Thus $\left({ }_{\bar{\theta}} A\right)(\Delta P)=A(\Delta P)$ as $k$-algebras. More precisely, for $Q$ as a subgroup of $P$ we get that

$$
\left({ }_{\bar{\theta}} A\right)(\Delta Q)=A(\Delta Q)
$$

as $C_{P}(Q)$-algebras. The $A$-module $M$ is still a ${ }_{\bar{\theta}} A$-module, and its restriction to $\mathcal{O} P$ via the structural homomorphism is now $\bar{\theta} M$. Thus, using $\S 3.3$, we get that $M(P, \theta)$ is an $A(\Delta P)$-module with a module structure which is induced by the $A^{\Delta P}$-module structure on $M^{(P, \theta)}$. In particular, if $A=\mathcal{O} G b$ for some finite group $G$ containing $P$ and some idempotent $b \in Z(\mathcal{O} G)$, it follows that $M(P, \theta)$ is a $k C_{G}(P) \operatorname{Br}_{\Delta P}(b)$-module.

3.7. By [4, Theorem 2.4], the generalised Brauer construction can be used to "lift" generalised decomposition maps. We describe this briefly. Let $G$ be a finite group and let $b$ be an idempotent in $Z(\mathcal{O} G)$. Denote by $\mathbb{Z} \operatorname{Irr}_{K}(G, b)$ the group of generalised characters of $G$ over $K$ associated with $b$ and by $\mathbb{Z I B r}_{K}(G, b)$ the corresponding group of generalised Brauer characters. If $b=1$ we write $\mathbb{Z} \operatorname{Irr}_{K}(G)$ instead of $\mathbb{Z I r r}_{K}(G, b)$. Denote by $\bar{b}$ the image of $b$ in $k G$. For a $k G \bar{b}$-module $N$ denote by $[[N]]$ its Brauer character viewed as an element in the group $\mathbb{Z I B r}_{K}(G, b)$ associated with $b$ (which can be identified with the Grothendieck group of $k G \bar{b}$-modules taken with respect to all short exact sequences). For $u$ a $p$-element in $G$ and $e$ an idempotent in $Z\left(k C_{G}(u)\right)$ the generalised decomposition map

$$
d_{(G, b)}^{(u, e)}: \mathbb{Z}_{\operatorname{Irr}_{K}}(G, b) \longrightarrow K \underset{\mathbb{Z}}{\otimes} \mathbb{Z I B r}_{K}\left(C_{G}(u), e\right)
$$

is defined by $d_{(G, b)}^{(u, e)}(\chi)(s)=\chi($ eus $)$ for all $p^{\prime}$-elements $s$ in $C_{G}(u)$. An $\mathcal{O} G$-module $M$ is called a linear source module if $M$ is a finite direct sum of summands of $\mathcal{O} G$ modules of the form $\mathcal{O} G \underset{\mathcal{O} Q}{\otimes}{ }_{\theta} \mathcal{O}$, where $Q$ runs over the $p$-subgroups of $G$ and $\theta$ over the group homomorphisms from $Q$ to $\mathcal{O}^{\times}$and where ${ }_{\theta} \mathcal{O}$ is the rank one module determined by $\theta$.

Theorem 3.8 ([4, Theorem 2.4]). Let $G$ be a finite group, let $u$ be a p-element in $G$, let $b$ be an idempotent in $Z(\mathcal{O} G)$ and let $e$ be an idempotent in $Z\left(k C_{G}(u)\right)$. Let $M$ be a linear source $\mathcal{O} G b$-module and let $\chi$ be the character of $M$. We have

$$
d_{(G, b)}^{(u, e)}(\chi)=\sum_{\theta \in \operatorname{Hom}(\langle u\rangle, \mathcal{O} \times)} \theta(u) \otimes[[M(\langle u\rangle, \theta)]] .
$$

The generalised Brauer construction "commutes" with "cutting by idempotents":

Lemma 3.9. Let $G$ be a finite group, let $P$ be a $p$-subgroup of $G$, let $\theta: P \rightarrow \mathcal{O}^{\times}$ be a group homomorphism and let $i$ be an idempotent in $(\mathcal{O} G)^{\Delta P}$ such that

$$
\operatorname{Br}_{\Delta P}(i) \neq 0 .
$$

For any $\mathcal{O} G$-module $M$ there is a natural isomorphism of $\operatorname{Br}_{\Delta P}(i) k C_{G}(P) \operatorname{Br}_{\Delta P}(i)$ modules

$$
(i M)(P, \theta) \cong \operatorname{Br}_{\Delta P}(i) M(P, \theta)
$$


Proof. The $k C_{G}(P)$-module structure on $M(P, \theta)$ is induced by the $(\mathcal{O} G)^{\Delta P}$-module structure on $M^{(P, \theta)}$. That is, for $a \in(\mathcal{O} G)^{\Delta P}$ and $m \in M^{(P, \theta)}$ we have

$$
a m+\operatorname{ker}\left(\operatorname{Br}_{(P, \theta)}^{M}\right)=\operatorname{Br}_{\Delta P}(a)\left(m+\operatorname{ker}\left(\operatorname{Br}_{(P, \theta)}^{M}\right)\right) .
$$

Since $i$ commutes with $P$ we have $(i M)^{(P, \theta)}=i\left(M^{(P, \theta)}\right)$. It follows that the map sending $i m+\operatorname{ker}\left(\operatorname{Br}_{(P, \theta)}^{M}\right)$ to $\operatorname{Br}_{\Delta P}(i)\left(m+\operatorname{ker}\left(\operatorname{Br}_{(P, \theta)}^{M}\right)\right.$ is in fact an equality $(i M)(P, \theta)=\operatorname{Br}_{\Delta P}(i) M(P, \theta)$ of subsets of $M(P, \theta)$.

3.10. Let $P$ be a finite $p$-group and let $A$ be an interior $P$-algebra. Following 21$]$ a local point of a subgroup $Q$ of $P$ on $A$ is an $\left(A^{\Delta Q}\right)^{\times}$-conjugacy class $\delta$ of primitive idempotents in $A^{\Delta Q}$ such that $\operatorname{Br}_{\Delta Q}(\delta) \neq 0$. Since $\operatorname{Br}_{\Delta Q}: A^{\Delta Q} \rightarrow A(\Delta Q)$ is a surjective algebra homomorphism, the standard lifting theorems for idempotents imply that then $\operatorname{Br}_{\Delta Q}(\delta)$ is a conjugacy class of primitive idempotents in $A(\Delta Q)$. If $Q, R$ are subgroups of $P, \delta$ a local point of $Q$ on $A$ and $\epsilon$ a local point of $R$ on $A$, we write $Q_{\delta} \subseteq R_{\epsilon}$ if $Q \subseteq R$ and if there are $i \in \delta, j \in \epsilon$ satisfying $i j=j i=i$. We refer to [28, for a detailed account on local pointed groups.

\section{Almost source algebras}

Let $G$ be a finite group and let $b$ be a block of $\mathcal{O} G$; that is, $b$ is a primitive idempotent in $Z(\mathcal{O} G)$. Let $P$ be a defect group of $b$; that is, $P$ is a maximal $p$-subgroup of $G$ with the property that $\operatorname{Br}_{\Delta P}(b) \neq 0$, where $\operatorname{Br}_{\Delta P}:(\mathcal{O} G)^{\Delta P} \rightarrow k C_{G}(P)$ is the Brauer homomorphism as described in the previous section. Equivalently, $P$ is a minimal subgroup of $G$ such that $b \in(\mathcal{O} G)_{P}^{G}$. Since $\operatorname{Br}_{\Delta P}(b) \neq 0$ there must be a primitive idempotent $i \in(\mathcal{O} G b)^{\Delta P}$ satisfying $\operatorname{Br}_{\Delta P}(i) \neq 0$. Such an idempotent $i$ is called a source idempotent, and the algebra $A=i \mathcal{O} G i$ is called a source algebra of $b$. This concept is due to Puig [21. A source algebra is always considered as an $i n$ terior $P$-algebra; that is, together with the group homomorphism $P \rightarrow A^{\times}$sending $u \in P$ to $u i=i u=i u i \in A^{\times}$. If $P^{\prime}$ is another defect group of $b$ and $i^{\prime} \in(\mathcal{O} G b)^{\Delta P^{\prime}}$ a source idempotent, then there is an $\mathcal{O}$-algebra isomorphism $\alpha: i \mathcal{O} G i \cong i^{\prime} \mathcal{O} G i^{\prime}$ and an element $x \in G$ such that ${ }^{x} P=P^{\prime}$ and such that $\alpha(u i)={ }^{x} u i^{\prime}$ for all $u \in P$. Source algebras of a block $b$ are Morita equivalent to the block algebra $\mathcal{O} G b$; more generally:

Proposition 4.1 ([21, 3.5]). Let $G$ be a finite group, let $b$ be a block of $\mathcal{O} G$, let $P$ be a defect group of $b$ and let $i$ be an idempotent in $(\mathcal{O} G b)^{\Delta P}$ such that $\operatorname{Br}_{\Delta P}(i) \neq 0$. The block algebra $\mathcal{O} G b$ and the algebra $i \mathcal{O} G i$ are Morita equivalent via the $\mathcal{O} G b$ $i \mathcal{O} G$-bimodule $\mathcal{O} G i$ and its $\mathcal{O}$-dual $i \mathcal{O} G$.

Proof. We sketch a proof for the convenience of the reader. The obvious map $i O G \otimes \mathcal{O} G i \rightarrow i \mathcal{O} G i$ given by multiplication in $\mathcal{O} G$ is clearly an isomorphism. $\mathcal{O} G$

For any idempotent $j \in(\mathcal{O} G b)^{\Delta P},(\mathcal{O} G j)_{\Delta P}^{\Delta G}$ is a left ideal, hence an ideal in the commutative algebra $Z(\mathcal{O} G b)$. Since $b \in(\mathcal{O} G b)_{\Delta P}^{\Delta G}$, Rosenberg's Lemma [28, (4.9)] implies that $b \in(\mathcal{O} G j)_{\Delta P}^{\Delta G}$ for some primitive idempotent $j \in(\mathcal{O} G b)^{\Delta P}$. Since $\operatorname{Br}_{\Delta P}(b) \neq 0$ this implies that $\operatorname{Br}_{\Delta P}(j) \neq 0$ and hence that $j$ belongs to a local point of $P$ on $\mathcal{O} G b$. Since all local points of $P$ on $\mathcal{O} G b$ are $N_{G}(P)$-conjugate, it follows that $b \in(\mathcal{O} G i)_{\Delta P}^{\Delta G}$. Thus there is $c \in(\mathcal{O} G)^{\Delta P}$ such that $\operatorname{Tr}_{\Delta P}^{\Delta G}(c i)=b$. Now $\operatorname{Tr}_{\Delta P}^{\Delta G}(c i)$ belongs to the two-sided ideal $\mathcal{O} G i \mathcal{O} G$ generated by $i$, and hence $b$ is in the image of the the map $\mathcal{O} G i \underset{i \mathcal{O} G i}{\otimes} i \mathcal{O} G \rightarrow \mathcal{O} G$ given by multiplication in $\mathcal{O} G$. Therefore this map is surjective and the result follows. 
Source algebras are known to be "relatively separable" with respect to their defect groups. As before, this is true in slightly greater generality:

Proposition 4.2. Let $G$ be a finite group, let $b$ be a block of $\mathcal{O} G$, let $P$ be a defect group of $b$ and let $i$ be an idempotent in $(\mathcal{O} G b)^{\Delta P}$ such that $\operatorname{Br}_{\Delta P}(i) \neq 0$. Set $A=i \mathcal{O} G i$. The canonical map $A \underset{\mathcal{O P}}{\otimes} A \rightarrow A$ induced by multiplication in $A$ splits as a homomorphism of $A$-A-bimodules; in particular, $A$ is isomorphic to a direct summand of $A \underset{\mathcal{O} P}{\otimes} A$ as an $A$-A-bimodule.

Proof. As in the proof of Proposition 4.1, write $b=\operatorname{Tr}_{\Delta P}^{\Delta G}(c i)$ for some $c \in(\mathcal{O} G)^{\Delta P}$. One checks that the map sending $a \in \mathcal{O} G$ to $\sum_{x \in[G / P]} a x c i \otimes i x^{-1}$ in $\mathcal{O} G i \underset{\mathcal{O} P}{\otimes} i \mathcal{O} G$ is a section for the canonical $\mathcal{O} G$-OOG-bimodule homomorphism $\mathcal{O} G i \otimes i \mathcal{O} G \rightarrow \mathcal{O} G b$ given by multiplication in $\mathcal{O} G$. Multiplying this homomorphism $\stackrel{\mathcal{O} G}{\text { by }} i$ on the left and right yields the required section as stated.

Note that the idempotent $i \in(\mathcal{O} G b)^{\Delta P}$ in Propositions 4.1 and 4.2 need not be primitive (if it is, then $A$ is a source algebra of $b$ ). A crucial property of a source idempotent $i \in(\mathcal{O} G b)^{\Delta P}$ is that, by [9, 1.8], for any subgroup $Q$ of $P$ the idempotent $\mathrm{Br}_{\Delta Q}(i)$ in $k C_{G}(Q)$ belongs in fact to a unique block algebra of $k C_{G}(Q)$. In the following definition we keep this key property but no longer require that $i$ be primitive:

Definition 4.3. Let $G$ be a finite group, let $b$ be a block of $\mathcal{O} G$ and let $P$ be a defect group of $b$. An idempotent $i$ in $(\mathcal{O} G b)^{\Delta P}$ is called an almost source idempotent if $\operatorname{Br}_{\Delta P}(i) \neq 0$ and for every subgroup $Q$ of $P$ there is a unique block $e_{Q}$ of $k C_{G}(Q)$ such that $\operatorname{Br}_{\Delta Q}(i) \in k C_{G}(Q) e_{Q}$. The interior $P$-algebra $i \mathcal{O} G i$ is then called an almost source algebra of the block $b$.

Remark 4.4. As pointed out before, any source idempotent is an almost source idempotent, by [9, 1.8]. The choice of an almost source idempotent $i \in(\mathcal{O} G b)^{\Delta P}$ determines a fusion system $\mathcal{F}$ as follows. For any subgroup $Q$ of $P$ denote by $e_{Q}$ the unique block of $k C_{G}(Q)$ such that $\operatorname{Br}_{\Delta Q}(i) e_{Q} \neq 0$. For any two subgroups $Q$, $R$ of $P$, the set $\operatorname{Hom}_{\mathcal{F}}(Q, R)$ is the set of all group homomorphisms $\varphi: Q \rightarrow R$ for which there is an element $x \in G$ satisfying $\varphi(u)=x u x^{-1}$ for all $u \in Q$ and satisfying $x e_{Q} x^{-1}=e_{x Q x^{-1}}$, which is equivalent to the inclusion of Brauer pairs ${ }^{x}\left(Q, e_{Q}\right) \subseteq\left(R, e_{R}\right)$ as defined in [1. It follows from the results in [1] that $\mathcal{F}$ is indeed a fusion system; see e.g. [18, §2] or [19] for more details. Moreover, a subgroup $Q$ of $P$ is fully $\mathcal{F}$-centralised if and only if $C_{P}(Q)$ is a defect group of the block $e_{Q}$ of $k C_{G}(Q)$. Given a subgroup $Q$ of $P$ it is always possible to find a subgroup $R$ of $P$ such that $Q \cong R$ in $\mathcal{F}$ and such that $R$ is fully $\mathcal{F}$-centralised. The following observation explains why we will need to work with fully centralised subgroups and almost source idempotents rather than source idempotents:

Proposition 4.5. Let $G$ be a finite group, let $b$ be a block of $\mathcal{O} G$, let $P$ be a defect group of $b$ and let $i$ be an almost source idempotent in $(\mathcal{O} G b)^{\Delta P}$. Let $\mathcal{F}$ be the fusion system determined by $i$ on $P$, let $Q$ be a fully $\mathcal{F}$-centralised subgroup of $P$ and let $e$ be the unique block of $k C_{G}(Q)$ such that $\operatorname{Br}_{\Delta Q}(i) e \neq 0$. The idempotent $\operatorname{Br}_{\Delta Q}(i)$ in $\left(k C_{G}(Q) e\right)^{\Delta C_{P}(Q)}$ is an almost source idempotent of the block e of $k C_{G}(Q)$.

Proof. Since $Q$ is fully $\mathcal{F}$-centralised, the group $C_{P}(Q)$ is a defect group of $e$ (cf. [18, §2]). The idempotent $\operatorname{Br}_{\Delta Q}(i)$ belongs to $k C_{G}(Q)^{C_{P}(Q)}$ and satisfies 
$\operatorname{Br}_{\Delta C_{P}(Q)}\left(\operatorname{Br}_{\Delta Q}(i)\right)=\operatorname{Br}_{\Delta Q C_{P}(Q)}(i)$ which is non-zero because even $\operatorname{Br}_{\Delta P}(i)$ is non-zero. Moreover, for every subgroup $S$ of $C_{P}(Q)$ we have $C_{C_{P}(Q)}(S)=$ $C_{P}(Q S)$ and $C_{C_{G}(Q)}(S)=C_{G}(Q S)$. Thus the unique block $f$ of $C_{G}(Q S)$ satisfying $\operatorname{Br}_{\Delta Q S}(i) f \neq 0$ is also the unique block of $k C_{C_{G}(Q)}(S)$ satisfying $\operatorname{Br}_{\Delta S}\left(\operatorname{Br}_{\Delta Q}(i)\right) f \neq$ 0 , and so $\operatorname{Br}_{\Delta Q}(i)$ is an almost source idempotent.

Proposition 4.6. Let $G$ be a finite group, let $b$ be a block of $\mathcal{O} G$, let $P$ be a defect group of $b$ and let $i$ be an almost source idempotent in $(\mathcal{O} G b)^{\Delta P}$. Set $A=i \mathcal{O} G i$ and let $\mathcal{F}$ be the fusion system of $A$ on $P$. Let $Q$ be a fully $\mathcal{F}$-centralised subgroup of $P$ and let $e$ be the unique block of $k C_{G}(Q)$ such that $\operatorname{Br}_{\Delta Q}(i) e \neq 0$.

(i) The block algebra $k C_{G}(Q) e_{Q}$ is Morita equivalent to the algebra $A(\Delta Q)$ via the bimodule $k C_{G}(Q) \mathrm{Br}_{\Delta Q}(i)$ and its dual.

(ii) For any local point $\delta$ of $Q$ on $\mathcal{O} G b$ satisfying $\operatorname{Br}_{\Delta Q}(\delta) e_{Q} \neq\{0\}$ we have $\delta \cap A \neq \emptyset$.

(iii) The canonical map $A(\Delta Q) \underset{k C_{P}(Q)}{\otimes} A(\Delta Q) \rightarrow A(\Delta Q)$ induced by multiplication in $A(\Delta Q)$ splits as a homomorphism of $A(\Delta Q)-A(\Delta Q)$-bimodules.

Proof. By Proposition 4.5, $\mathrm{Br}_{\Delta Q}(i)$ is an almost source idempotent, and thus Proposition 4.1 applies to $k C_{G}(Q) e$ and $\operatorname{Br}_{\Delta Q}(i)$ which implies statement (i). If $\delta$ is a local point of $Q$ on $\mathcal{O} G b$ satisfying $\operatorname{Br}_{\Delta Q}\left(e_{Q}\right) \neq\{0\}$, then $\operatorname{Br}_{\Delta Q}(\delta)$ is a conjugacy class of primitive idempotents in $k C_{G}(Q) e$. Since $k C_{G}(Q) e$ is Morita equivalent to $A(\Delta Q)=\operatorname{Br}_{\Delta Q}(i) k C_{G}(Q) \mathrm{Br}_{\Delta Q}(i)$ there is $j \in \delta$ such that $\operatorname{Br}_{\Delta Q}(j) \in \operatorname{Br}_{\Delta Q}(i) k C_{G}(Q) \operatorname{Br}_{\Delta Q}(i)$. The usual lifting theorems for idempotents imply that $j$ can be chosen in $A^{\Delta Q}=i(k G)^{\Delta Q} i$, whence statement (ii). Statement (iii) follows from Proposition 4.2 applied to $k C_{G}(Q), e_{Q}, \operatorname{Br}_{\Delta Q}(i)$ and $C_{P}(Q)$ instead of $\mathcal{O} G, b, i$ and $P$, respectively.

This explains why it is technically easier to work at the source algebra level: while $(\mathcal{O} G b)(\Delta Q)=k C_{G}(Q) \operatorname{Br}_{\Delta Q}(b)$ may decompose as a product of more than one block algebra, the algebra $A(\Delta Q)$ remains indecomposable so long as one chooses $Q$ to be fully centralised with respect to the fusion system of $A$ on $P$. The following result is a tool to switch back and forth between block and source algebras:

Proposition 4.7. Let $G$ be a finite group, let $b$ be a block of $\mathcal{O} G$, let $P$ be a defect group of $b$ and let $i$ be an almost source idempotent in $(\mathcal{O} G b)^{\Delta P}$. Set $A=i \mathcal{O} G i$ and let $\mathcal{F}$ be the fusion system of $A$ on $P$. Let $Q$ be a fully $\mathcal{F}$-centralised subgroup of $P$ and let $e$ be the unique block of $k C_{G}(Q)$ such that $\operatorname{Br}_{\Delta Q}(i) e \neq 0$. Let $\theta: Q \rightarrow \mathcal{O}^{\times}$ be a group homomorphism. For any A-module $M$ there is a natural isomorphism of $k C_{G}(Q) e$-modules

$$
e((\mathcal{O} G i \underset{A}{\otimes} M)(Q, \theta)) \cong k C_{G}(Q) \operatorname{Br}_{\Delta Q}(i) \underset{A(\Delta Q)}{\otimes} M(Q, \theta) .
$$

Proof. Since $\mathrm{Br}_{\Delta Q}(i) \in k C_{G}(Q) e$, both sides in the statement are indeed $k C_{G}(Q) e$ modules. By Proposition 4.6(i), multiplication by $\operatorname{Br}_{\Delta Q}(i)$ induces a Morita equivalence between $k C_{G}(Q) e$ and $A(\Delta Q)$. Thus, in order to show that there is a natural isomorphism as stated, we must first multiply both sides with $\operatorname{Br}_{\Delta Q}(i)$. Using Lemma 3.9, the left side becomes

$$
\operatorname{Br}_{\Delta Q}(i)(k G i \underset{A}{\otimes} M)(Q, \theta)=(i k G i \underset{A}{\otimes} M)(Q, \theta)=(A \underset{A}{\otimes} M)(Q, \theta) \cong M(Q, \theta)
$$


and the right side becomes

$$
\operatorname{Br}_{\Delta Q}(i) k C_{G}(Q) \operatorname{Br}_{\Delta Q}(i) \underset{A(\Delta Q)}{\otimes} M(Q, \theta) \cong M(Q, \theta) ;
$$

both sides are naturally isomorphic as $A(\Delta Q)$-modules.

Proposition 4.8. Let $G$ be a finite group, $b$ a block of $\mathcal{O} G, P$ a defect group of $b$ and $i \in(\mathcal{O} G b)^{\Delta P}$ an almost source idempotent. Let $Q$ be a subgroup of $P$ and let $e, e^{\prime}$ be two different blocks of $k_{G}(Q)$. For any subgroup $R$ of $P$ and any direct summand $Y$ of $\mathcal{O} G i \underset{\mathcal{O} R}{\otimes} i \mathcal{O} G$ we have $\mathrm{e} Y(\Delta Q) e^{\prime}=\{0\}$.

Proof. We may assume $\mathcal{O}=k$. Since $i$ is an almost source idempotent, for any subgroup $S$ of $P$ there is a unique block $e_{S}$ of $k C_{G}(S)$ satisfying $\operatorname{Br}_{\Delta S}(i) e_{S} \neq 0$. The image of the set $G \times G$ in $k G \otimes k G$ is a $k$-basis which is stable under the action of $\Delta Q$. In order to compute $Y(\Delta Q)$ we have to determine the $\Delta Q$-fixpoints in this basis. For $x, y \in G$ the image $x \otimes y$ in $k G \underset{k R}{\otimes} k G$ is fixed under the action of $\Delta Q$ if for every $u \in Q$ there is $r_{u} \in R$ such that $u x=x r_{u}$ and $y u^{-1}=\left(r_{u}\right)^{-1} y$, or equivalently, if and only if $x y \in C_{G}(Q)$ and $Q^{x}={ }^{y} Q \subseteq R$. Thus we have

$$
(k G \underset{k R}{\otimes} k G)^{\Delta Q}=\sum_{(x, y)} k(x \otimes y) \oplus \operatorname{ker}\left(\operatorname{Br}_{\Delta Q}\right),
$$

where $(x, y)$ runs over the set of pairs in $G \times G$ satisfying $x y \in C_{G}(Q)$ and $Q^{x} \subseteq R$. Consequently, we get that

$$
\left(e k G i \underset{k R}{\otimes} i k G e^{\prime}\right)^{\Delta Q}=\sum_{(x, y)} k\left(e x i \otimes i y e^{\prime}\right)+\operatorname{ker}\left(\operatorname{Br}_{\Delta Q}\right)
$$

with $(x, y)$ as before. Write $e x i=e\left({ }^{x} i\right) x$. Since $i$ is an almost source idempotent we have $e\left({ }^{x} i\right) \in \operatorname{ker}\left(\operatorname{Br}_{\Delta Q}\right)$ unless $e={ }^{x}\left(e_{Q^{x}}\right)$. Similarly, we have $\left({ }^{y} i\right) e^{\prime} \in \operatorname{ker}\left(\operatorname{Br}_{\Delta Q}\right)$ unless $e^{\prime}=\left(e_{y_{Q}}\right)^{y}$. But these two equalities would imply the contradiction $e=e^{\prime}$ since $x y \in C_{G}(Q)$. Thus at least one of $e\left({ }^{x} i\right),\left({ }^{y} i\right) e^{\prime}$ is contained in $\operatorname{ker}\left(\operatorname{Br}_{\Delta Q}\right)$, whence the result.

Proposition 4.9. Let $G, H$ be finite groups and let b, c be blocks of $\mathcal{O} G, \mathcal{O} H$ with defect group $P, Q$, respectively. Let $i \in(\mathcal{O} G b)^{\Delta P}$ and $j \in(\mathcal{O H} c)^{\Delta Q}$ be almost source idempotents, let $\mathcal{F}$ be the fusion system on $P$ determined by $i$ and let $\mathcal{G}$ be the fusion system on $Q$ determined by $j$. Identify $\mathcal{O} G \underset{\mathcal{O}}{\otimes} \mathcal{O} H$ and $\mathcal{O}(G \times H)$ through the canonical isomorphism.

(i) $b \otimes c$ is a block of $\mathcal{O}(G \times H)$, and $P \times Q$ is a defect group of $b \otimes c$.

(ii) $i \otimes j$ is an almost source idempotent of $b \otimes c$.

(iii) The fusion system determined by $i \otimes j$ on $P \times Q$ is equal to $\mathcal{F} \times \mathcal{G}$.

Proof. Statement (i) is well known (and easy to prove). For $R$ a subgroup of $P \times Q$, denote by $R_{1}$ the image of $R$ under the projection $P \times Q \rightarrow P$ and by $R_{2}$ the image of $R$ under the projection $P \times Q \rightarrow Q$. Then $R \subseteq R_{1} \times R_{2}$ and $C_{G \times H}(R)=$ $C_{G}\left(R_{1}\right) \times C_{H}\left(R_{2}\right)$. Thus $\operatorname{Br}_{\Delta R}(i \otimes j)=\operatorname{Br}_{\Delta R_{1}}(i) \otimes \operatorname{Br}_{\Delta R_{2}}(j)$. Therefore, if $e_{1}, f_{2}$ are the unique blocks of $k C_{G}\left(R_{1}\right), k C_{H}\left(R_{2}\right)$, respectively, satisfying $\operatorname{Br}_{\Delta R_{1}}(i) e_{1} \neq 0$ and $\operatorname{Br}_{\Delta R_{2}}(j) f_{2} \neq 0$, then $e=e_{1} \otimes f_{2}$ is the unique block of $C_{G \times H}(R)$ satisfying $\operatorname{Br}_{\Delta R}(i \otimes j) e \neq 0$. This shows (ii), and (iii) is clear. 
Proposition 4.10. Let $G$ be a finite group, let $b$ be a block of $\mathcal{O} G$, let $P$ be a defect group of $b$ and let $i \in(\mathcal{O} G b)^{\Delta P}$ be an almost source idempotent. Let $\alpha$ be the anti-automorphism of $\mathcal{O} G$ sending $x \in G$ to $x^{-1}$. Set $b^{0}=\alpha(b)$ and $i^{0}=\alpha(i)$. Then $b^{0}$ is a block of $\mathcal{O} G$ having $P$ as a defect group and $i^{0} \in\left(\mathcal{O} G b^{0}\right)^{\Delta P}$ is an almost source idempotent of $b^{0}$. Moreover, the fusion systems on $P$ determined by $i$ and by $i^{0}$ are equal.

Proof. Straightforward verification.

Combining Propositions 4.7 and 4.9, Proposition 4.10 yields another statement about switching between block algebras and source algebras for bimodules which will be needed in the proof of Theorem 1.4:

Proposition 4.11. Let $G, H$ be finite groups and let b, $c$ be blocks of $\mathcal{O} G, \mathcal{O} H$, respectively, having a common defect group $P$. Let $i \in(\mathcal{O} G b)^{\Delta P}$ and $j \in(\mathcal{O H} H)^{\Delta P}$ be almost source idempotents. Suppose that $i$ and $j$ determine the same fusion system $\mathcal{F}$ on $P$. Let $Q$ be a fully $\mathcal{F}$-centralised subgroup of $P$ and let $e$ and $f$ be the unique blocks of $k C_{G}(Q)$ and $k C_{H}(Q)$, respectively, satisfying $\operatorname{Br}_{\Delta Q}(i) e \neq 0$ and $\operatorname{Br}_{\Delta Q}(j) f \neq 0$. Let $X$ be an $A$-B-bimodule and set $Y=e((\mathcal{O} G i \underset{A}{\otimes} \underset{B}{\otimes} j \mathcal{O} H)(\Delta Q)) f$. Then

$$
Y \cong k C_{G}(Q) \operatorname{Br}_{\Delta Q}(i) \underset{A(\Delta Q)}{\otimes} X(\Delta Q) \underset{B(\Delta Q)}{\otimes} \operatorname{Br}_{\Delta Q}(j) k C_{H}(Q)
$$

as $k C_{G}(Q) e-k C_{H}(Q) f$-bimodules and

$$
\operatorname{Br}_{\Delta Q}(i) Y \operatorname{Br}_{\Delta Q}(j) \cong X(\Delta Q)
$$

as $A(\Delta Q)-B(\Delta Q)$-bimodules.

Proof. By Proposition $2.2, \Delta Q$ is fully $\mathcal{F} \times \mathcal{F}$-centralised. By Proposition 4.7 applied to $G \times H$, the almost source idempotent $i \otimes j^{0}$, the subgroup $\Delta Q$ of $P \times P$ and the block $e \otimes f^{0}$ of $C_{G \times H}(\Delta Q)$ we get the first of the two isomorphisms. Multiplying this on the left and on the right by $\operatorname{Br}_{\Delta Q}(i)$ and $\operatorname{Br}_{\Delta Q}(j)$, respectively, yields the second isomorphism.

\section{Fusion in Almost source Algebras}

By [22, 3.1], the fusion system $\mathcal{F}$ of a block $b$ of $\mathcal{O} G$ can be read from the $\mathcal{O} P$-O $P$-bimodule structure of the source algebra $A=i \mathcal{O} G i$ of $b$. The following propositions collect some technical details around this theme, similar to material in [15, 17, extended here to almost source idempotents.

Proposition 5.1. Let $G$ be a finite group, let $b$ be a block of $\mathcal{O} G$ with defect group $P$, let $i \in(\mathcal{O} G b)^{\Delta P}$ be an almost source idempotent and set $A=i \mathcal{O} G i$. Denote by $\mathcal{F}$ the fusion system of $A$ on $P$. Let $Q$ be a fully $\mathcal{F}$-centralised subgroup of $P$ and let $\varphi: Q \rightarrow P$ be a morphism in $\mathcal{F}$. Set $R=\varphi(Q)$. Denote by $e_{Q}, e_{R}$ the unique blocks of $k C_{G}(Q), k C_{G}(R)$ satisfying $\mathrm{Br}_{\Delta Q}(i) e_{Q} \neq 0$ and $\mathrm{Br}_{\Delta R}(i) e_{R} \neq 0$.

(i) For any primitive idempotent $n$ in $(\mathcal{O} G b)^{\Delta R}$ satisfying $\operatorname{Br}_{\Delta R}(n) e_{R} \neq 0$ there is a primitive idempotent $m$ in $A^{\Delta Q}$ satisfying $\operatorname{Br}_{\Delta Q}(m) \neq 0$ such that $m \mathcal{O} G \cong{ }_{\varphi}(n \mathcal{O} G)$ as $\mathcal{O} Q-\mathcal{O} G b$-bimodules and such that $\mathcal{O} G m \cong(\mathcal{O} G n)_{\varphi}$ as $\mathcal{O} G b$-OQ $Q$-bimodules.

(ii) For any primitive idempotent $n$ in $A^{\Delta R}$ satisfying $\operatorname{Br}_{\Delta R}(n) \neq 0$ there is a primitive idempotent $m$ in $A^{\Delta Q}$ satisfying $\operatorname{Br}_{\Delta Q}(m) \neq 0$ such that $m A \cong$ ${ }_{\varphi}(n A)$ as $\mathcal{O} Q$ - $A$-bimodules and such that $A m \cong(A n)_{\varphi}$ as $A$-O $\mathcal{O}$-bimodules. 
Proof. Since $\varphi$ belongs to the fusion system $\mathcal{F}$ there is an element $x \in G$ such that $\varphi(u)=x u x^{-1}$ for all $u \in Q$ and such that ${ }^{x}\left(e_{Q}\right)=e_{R}$. Let $n$ be a primitive idempotent in $(\mathcal{O} G b)^{\Delta R}$ satisfying $\operatorname{Br}_{\Delta R}(n) e_{R} \neq 0$. Denote by $\nu$ the local point of $R$ on $\mathcal{O} G b$ containing $n$. Since ${ }^{x} Q=R$ there is a local point $\mu$ of $Q$ on $\mathcal{O} G b$ such that $\nu={ }^{x} \mu$. Since $\operatorname{Br}_{\Delta R}(\nu) e_{R} \neq 0$ we have $\operatorname{Br}_{\Delta Q}(\mu) e_{Q} \neq 0$. This implies that $\operatorname{Br}_{\Delta Q}(\mu)$ is in fact a conjugacy class of primitive idempotents in $k C_{G}(Q) e_{Q}$. Now $Q$ is fully $\mathcal{F}$-centralised, and hence by Proposition $4.6(i i), \mu$ must contain an element $m$ such that $m \in A$. Moreover, $n^{x}$ and $m$ both belong to the same point $\mu$ of $Q$ on $\mathcal{O} G b$, and hence there is an element $c \in\left((\mathcal{O} G b)^{\Delta Q}\right)^{\times}$such that ${ }^{x c} m=$ $n$. The map sending $m a \in m \mathcal{O} G$ to $x c m a=n x c a$ is the required isomorphism $m \mathcal{O} G \cong{ }_{\varphi}(n \mathcal{O} G)$. The map sending $a m$ to $a m c^{-1} x^{-1}=a c^{-1} x^{-1} n$ is the required isomorphism $\mathcal{O} G m \cong(\mathcal{O} G n)_{\varphi}$. This proves (i). Statement (ii) is played back to (i) via the Morita equivalence between $\mathcal{O} G b$ and $A$. Let $n$ be a primitive idempotent in $A^{\Delta R}$ satisfying $\operatorname{Br}_{\Delta R}(n) \neq 0$. The unit element $i$ of $A$ satisfies $\operatorname{Br}_{\Delta R}(i) e_{R}=$ $\operatorname{Br}_{\Delta R}(i)$ by the uniqueness of the inclusion of Brauer pairs. Thus $\operatorname{Br}_{\Delta R}(n) e_{R} \neq$ 0 . Therefore statement (i) applies and yields a primitive idempotent $m \in A^{\Delta Q}$ satisfying $\operatorname{Br}_{\Delta Q}(m) \neq 0$ such that there are isomorphisms $m \mathcal{O} G \cong{ }_{\varphi}(n \mathcal{O} G)$ and $\mathcal{O} G m \cong(\mathcal{O} G n)_{\varphi}$. Multiplying these isomorphisms by $i$ on the right and on the left, respectively, yields the isomorphisms as stated in (ii).

The following is [17, 7.7, 7.8] generalised to almost source idempotents.

Proposition 5.2. Let $G$ be a finite group, let $b$ be a block of $\mathcal{O} G$ with defect group $P$, let $i$ be an almost source idempotent in $(\mathcal{O} G b)^{\Delta P}$ and set $A=i \mathcal{O} G i$. Denote by $\mathcal{F}$ be the fusion system of $A$ on $P$. Let $Q, R$ be subgroups of $P$.

(i) Every indecomposable direct summand of $A$ as an $\mathcal{O} Q-\mathcal{O} R$-bimodule is isomorphic to $\mathcal{O} Q \otimes_{\mathcal{O} S}{ }_{\varphi} \mathcal{O} R$ for some subgroup $S$ of $Q$ and some morphism $\varphi: S \rightarrow R$ belonging to $\mathcal{F}$.

(ii) If $\varphi: Q \rightarrow R$ is an isomorphism in $\mathcal{F}$ such that $R$ is fully $\mathcal{F}$-centralised, then ${ }_{\varphi} \mathcal{O}$ is isomorphic to a direct summand of $A$ as an $\mathcal{O} Q-\mathcal{O} R$-bimodule.

Proof. (i) Let $Y$ be an indecomposable direct summand of $A$ as an $\mathcal{O} Q-\mathcal{O} R$-bimodule. Then $Y$ has a $Q \times R$-stable $\mathcal{O}$-basis on which $Q$ and $R$ act freely on the left and on the right, respectively. Thus

$$
Y \cong \mathcal{O} Q \underset{\mathcal{O} S}{\otimes} \varphi \mathcal{O} R
$$

for some subgroup $S$ of $Q$ and some injective group homomorphism $\varphi: S \rightarrow R$. Set $T=\varphi(S)$. Restricting $Y$ to $S \times T$ shows that ${ }_{\varphi} \mathcal{O} T$ is isomorphic to a direct summand of $Y$, hence of $A$, as an $\mathcal{O} S$ - $\mathcal{O} T$-bimodule. Now $A$ is a direct summand of $\mathcal{O} G$ as an $\mathcal{O} P-\mathcal{O} P$-bimodule. In particular, ${ }_{\varphi} \mathcal{O} T$ is isomorphic to a direct summand of $\mathcal{O} G$ as an $\mathcal{O} S$ - $\mathcal{O} T$-bimodule, hence isomorphic to $\mathcal{O} S y^{-1}=y^{-1} \mathcal{O} T$ for some element $y \in G$ such that ${ }^{y} S=T$ and such that ${ }^{y} s=\varphi(s)$ for all $s \in S$. Then $\mathcal{O} S$ is isomorphic to a direct summand of $i \mathcal{O} G i y=i \mathcal{O} G y^{-1} i y$ as an $\mathcal{O} S$-O $S$-bimodule. Thus $\operatorname{Br}_{\Delta S}\left(i \mathcal{O} G y^{-1} i y\right) \neq 0$ by Lemma 3.5. Since $\operatorname{Br}_{\Delta S}(i) \in k C_{G}(S) e_{S}$ this also forces that $\operatorname{Br}_{\Delta S}\left(y^{-1} i y\right) e_{S} \neq 0$. Conjugating by $y$ yields that $\operatorname{Br}_{\Delta T}(i)^{y} e_{S} \neq 0$. But then necessarily ${ }^{y} e_{S}=e_{T}$ because $e_{T}$ is the unique block of $k C_{G}(T)$ with the property $\operatorname{Br}_{\Delta T}(i) e_{T} \neq 0$. This shows that $\varphi$ is a morphism in the fusion system $\mathcal{F}$, whence (i).

(ii) Since $\varphi$ belongs to $\mathcal{F}$ there is an element $x \in G$ such that $\varphi(u)={ }^{x} u$ for all $u \in Q$ and such that ${ }^{x} e_{Q}=e_{R}$. Let $\mu$ be a local point of $Q$ on $\mathcal{O} G b$ such 
that $\mu \cap A \neq \emptyset$. Set $\nu={ }^{x} \mu$; that is, $\nu$ is the local point of $R$ on $\mathcal{O} G b$ such that ${ }^{x}\left(Q_{\delta}\right)=R_{\nu}$. Since $\mu \cap A \neq \emptyset$ we have $\operatorname{Br}_{\Delta Q}(\mu) e_{Q} \neq 0$. Conjugating by $x$ implies that $\operatorname{Br}_{\Delta R}(\nu) e_{R} \neq 0$. Since $R$ is fully $\mathcal{F}$-centralised, it follows from Proposition 4.6(ii) that also $\nu \cap A \neq \emptyset$. Let $m \in \mu \cap A$ and let $n \in \nu \cap A$. Note that $n$ and ${ }^{x} m$ both belong to $\nu$; hence are conjugate in $\left(A^{R}\right)^{\times}$. Since $\operatorname{Br}_{\Delta Q}(m) \neq 0$ we get $(m \mathcal{O} G m)(\Delta Q) \neq\{0\}$; hence $m \mathcal{O} G m$ has a direct summand isomorphic to $\mathcal{O} Q$ as an $\mathcal{O} Q-\mathcal{O} Q$-bimodule. Therefore, $m \mathcal{O} G m x^{-1}=m \mathcal{O} G x m x^{-1} \cong m \mathcal{O} G n=m A n$ has a direct summand isomorphic to $\mathcal{O} Q_{\varphi^{-1}} \cong{ }_{\varphi} \mathcal{O} R$ as an $\mathcal{O} Q$ - $\mathcal{O} R$-bimodule.

Corollary 5.3. With the notation of Proposition 5.2, the fusion system $\mathcal{F}$ is generated by the set of inclusions between subgroups of $P$ and automorphisms $\varphi$ of any subgroup $Q$ of $P$ for which ${ }_{\varphi} O Q$ is isomorphic to a direct summand of $A$ as an $\mathcal{O} Q-\mathcal{O} Q$-bimodule.

Proof. This follows from Proposition 5.2 and Alperin's fusion theorem.

\section{Vertices of $p$-PERMUtation modules FOR ALMOST SOURCE ALGEBRAS}

The Brauer construction sends $p$-permutation modules to $p$-permutation modules. Combining material from the previous sections we can be more precise regarding vertices:

Proposition 6.1. Let $A$ be an almost source algebra of a block with defect group $P$ and fusion system $\mathcal{F}$. Let $R$ be a subgroup of $P$ and let $X$ be an indecomposable direct summand of the $A$-module $A \underset{\mathcal{O} R}{\otimes} \mathcal{O}$. Then $X$ is isomorphic to a direct summand of $A m \underset{\mathcal{O} Q}{\otimes} \mathcal{O}$ for some fully $\mathcal{F}$-centralised subgroup $Q$ of $P$ which is isomorphic, in $\mathcal{F}$, to a subgroup of $R$ and some primitive idempotent $m \in A^{\Delta Q}$ satisfying $\operatorname{Br}_{\Delta Q}(m) \neq 0$.

Proof. Since $X$ is indecomposable, $X$ is isomorphic to a direct summand of $A n \otimes \mathcal{O}$ for some primitive idempotent $n \in A^{\Delta R}$. Up to replacing $R$ by a proper subgroup we may assume by [15, 2.6] that $\operatorname{Br}_{\Delta R}(n) \neq 0$. Let $Q$ be a fully $\mathcal{F}$-centralised subgroup of $P$ such that there is an isomorphism $\varphi: Q \cong R$ in $\mathcal{F}$. By Proposition 5.1(ii) there is a primitive idempotent $m \in A^{\Delta Q}$ such that $\operatorname{Br}_{\Delta Q}(m) \neq 0$ and such that $A m \cong(A n)_{\varphi}$ as $A$-OOQ-bimodules. Then $A n \underset{\mathcal{O} R}{\otimes} \mathcal{O} \cong A m \underset{\mathcal{O} Q}{\otimes} \mathcal{O}$, and hence in particular, $X$ is isomorphic to a direct summand of $A \underset{\mathcal{O Q}}{\otimes} \mathcal{O}$ as stated.

We use Proposition 6.1 to track vertices of indecomposable summands of $p$ permutation modules after applying the Brauer construction:

Theorem 6.2. Let $A$ be an almost source algebra of a block with defect group $P$ and fusion system $\mathcal{F}$. Let $Q, R$ be subgroups of $P$ such that $Q$ is fully $\mathcal{F}$-centralised. Let $Y$ be an indecomposable direct summand of the $A(\Delta Q)$-module $(A \underset{\mathcal{O} R}{\otimes} \mathcal{O})(Q)$. Then $Y$ is isomorphic to a direct summand of $A(\Delta Q) m \otimes k$ for some subgroup $S$ of $C_{P}(Q)$ containing $Z(Q)$ and some primitive idempotent $m \in A(\Delta Q)^{\Delta S}$ satisfying $\operatorname{Br}_{\Delta S}(m) \neq 0$ such that $S$ is fully $C_{\mathcal{F}}(Q)$-centralised and $Q S$ is isomorphic in $\mathcal{F}$ to a subgroup of $R$. 
Proof. By Proposition 4.6, $A(\Delta Q)$ is isomorphic to a direct summand of $A(\Delta Q) \underset{k C_{P}(Q)}{\otimes} A(\Delta Q)$. Tensoring with $-\underset{A(\Delta Q)}{\otimes} Y$ implies that $Y$ is isomorphic to a direct summand of $A(\Delta Q) \underset{k C_{P}(Q)}{\otimes} Y$. Since $Y$ is indecomposable, $Y$ is in fact isomorphic to a direct summand of $A(\Delta Q) \underset{k C_{P}(Q)}{\otimes} W$ for some indecomposable direct summand of $Y$ as a $k C_{P}(Q)$-module. Thus we need to determine the $k C_{P}(Q)$-module structure of $Y$. Let $X$ be a $P$ - $P$-stable $\mathcal{O}$-basis of $A$. We use the notation $X \otimes 1$ for the image of the set $X$ in $A \underset{\mathcal{O} R}{\otimes} \mathcal{O}$. The set $X \otimes 1$ is a $P$-stable $\mathcal{O}$-basis of $A \underset{\mathcal{O} R}{\otimes} \mathcal{O}$. Thus, in order to compute $(A \underset{\mathcal{O} R}{\otimes} \mathcal{O})(Q)$ we need to determine the $Q$-fixpoints in the set $X \otimes 1$. For $x, y \in X$ the images $x \otimes 1$ and $y \otimes 1$ in $X \otimes 1$ are equal if and only if there is an element $r \in R$ such that $y=x r$. Therefore, $x \otimes 1 \in(X \otimes 1)^{Q}$ if and only if for every $u \in Q$ there is $r_{u} \in R$ such that $u x=x r_{u}$. In that case, $r_{u}$ is then uniquely determined by $u$ because $P$ acts freely on the right of $X$. Since $P$ also acts freely on the left of $X$, the map sending $u \in Q$ to $r_{u} \in R$ is an injective group homomorphism $\varphi: Q \rightarrow R$. Set

$$
U_{\varphi}=\{x \in X \mid u x=x \varphi(u)(\forall u \in Q)\} .
$$

Note that by Proposition 5.2(i) any $\varphi$ arising in this way belongs to the fusion system $\mathcal{F}$ because $\mathcal{O} Q x=x \mathcal{O} \varphi(Q) \cong{ }_{\varphi} \mathcal{O} \varphi(Q)$ is a direct summand of $A$ as an $\mathcal{O} Q-\mathcal{O} \varphi(Q)$-bimodule. It follows from the above that

$$
(X \otimes 1)^{Q}=\bigcup_{\varphi} U_{\varphi} \otimes 1,
$$

where $\varphi$ runs over the set $\operatorname{Hom}_{\mathcal{F}}(Q, R)$. Let $\varphi, \psi \in \operatorname{Hom}_{\mathcal{F}}(Q, R)$ and denote by $\tilde{\varphi}$, $\tilde{\psi}$ their images in the orbit space $\operatorname{Inn}(R) \backslash \operatorname{Hom}_{\mathcal{F}}(Q, R)$, where the group of inner automorphisms $\operatorname{Inn}(R)$ of $R$ acts by composition of group homomorphisms on the set $\operatorname{Hom}_{\mathcal{F}}(Q, R)$. Suppose there is an element $x \in U_{\varphi}$ and $y \in U_{\psi}$ such that $x \otimes 1=y \otimes 1$. Then there is an element $r \in R$ such that $y=x r$, and for all $u \in U$, we have $u x=x \varphi(u)$ and $u y=y \psi(u)$. Thus $u x r=x \varphi(u) r=x r \psi(u)$; hence $\varphi(u) r=\psi(u) r$, or equivalently, $\varphi(u)={ }^{r} \psi(u)$ for all $u \in Q$. This means that $\tilde{\varphi}=\tilde{\psi}$. It follows that $U_{\varphi} \otimes 1=U_{\psi} \otimes 1$ if $\tilde{\varphi}=\tilde{\psi}$ and that $U_{\varphi} \otimes 1 \cap U_{\psi} \otimes 1=\emptyset$ if $\tilde{\varphi} \neq \tilde{\psi}$. In other words, $(X \otimes 1)^{Q}$ is in fact the disjoint union

$$
(X \otimes 1)^{Q}=\bigcup_{\varphi} U_{\varphi} \otimes 1
$$

with $\varphi$ running over a set of representatives in $\operatorname{Hom}_{\mathcal{F}}(Q, R)$ of $\operatorname{Inn}(R) \backslash \operatorname{Hom}_{\mathcal{F}}(Q, R)$. Therefore, by Lemma 3.4, we have

$$
(A \underset{\mathcal{O} R}{\otimes} \mathcal{O})(Q) \cong k\left((X \otimes 1)^{Q}\right)=\bigoplus_{\varphi} k\left(U_{\varphi} \otimes 1\right)
$$

with $\varphi$ running as before over a set of representatives in $\operatorname{Hom}_{\mathcal{F}}(Q, R)$ of $\operatorname{Inn}(R) \backslash$ $\operatorname{Hom}_{\mathcal{F}}(Q, R)$. This is a decomposition of $(A \underset{\mathcal{O} R}{\otimes} \mathcal{O})(Q)$ as a $k C_{P}(Q)$-module because the subsets $U_{\varphi}$ of $X$ are invariant under the left action of $C_{P}(Q)$. Thus the direct summand $W$ of $Y$ as a $k C_{P}(Q)$-module is isomorphic to a direct summand of $k\left(U_{\varphi} \otimes 1\right)$ for some $\varphi \in \operatorname{Hom}_{\mathcal{F}}(Q, R)$. Now $W$ is a permutation $k C_{P}(Q)$-module, 
and hence, in order to determine its structure, it suffices to determine the stabilisers in $C_{P}(Q)$ of basis elements $x \otimes 1 \in U_{\varphi} \otimes 1$. Let $x, y \in U_{\varphi}$ such that $x \otimes 1=y \otimes 1$. Then, on one hand, $y=x r$ for some $r \in R$, and on the other hand, $u x=x \varphi(u)$ and $u y=y \varphi(u)$ for all $u \in Q$. Thus $u x r=x \varphi(u) r=x r \varphi(u)$, which implies $r \in C_{P}(\varphi(Q))$. It follows that the stabiliser $S$ in $C_{P}(Q)$ of an element $x \otimes 1 \in U_{\varphi} \otimes 1$ consists of all elements $z \in C_{P}(Q)$ such that $z x=x r_{z}$ for some $r_{z} \in C_{P}(\varphi(Q))$. In particular $Z(Q) \subseteq S$ because $z x=x \varphi(z)$ for $z \in Z(Q)$. It follows that there is a well-defined group homomorphism $\psi: Q S \longrightarrow R$ given by $\psi(u z)=\varphi(u) r_{z}$ for all $u \in Q$ and $z \in S$. Note that $\psi$ has the property $u z x=x \psi(u z)$, so $\mathcal{O}(Q S) x=$ $x \mathcal{O}(\psi(Q S)) \cong{ }_{\psi} \mathcal{O} \psi(Q S)$ is a direct summand of $A$ as an $\mathcal{O}(Q S)-\mathcal{O} \psi(Q S)$-bimodule. Hence $\psi$ belongs to the fusion system $\mathcal{F}$ by Proposition 5.2(i). This shows that $Y$ is isomorphic to a direct summand of $A(\Delta Q) \underset{k S}{\otimes} k$ for some subgroup $S$ of $C_{P}(Q)$ containing $Z(Q)$ for which there exists a morphism $\psi: Q S \rightarrow R$ in $\mathcal{F}$. We need to show that $S$ can furthermore be chosen to be fully $C_{\mathcal{F}}(Q)$-centralised. Applying Proposition 6.1 to $A(\Delta Q)$ and its fusion system, $C_{\mathcal{F}}(Q)$ shows that $Y$ is isomorphic to a direct summand of $A(\Delta Q) m \otimes k$ for some fully $C_{\mathcal{F}}(Q)$-centralised subgroup $T$ of $C_{P}(Q)$ for which there exists a morphism $\tau: T \rightarrow S$ in $C_{\mathcal{F}}(Q)$ and some primitive idempotent $m \in A(\Delta Q)^{\Delta T}$ satisfying $\operatorname{Br}_{\Delta T}(m) \neq 0$. By the definition of $C_{\mathcal{F}}(Q)$, the morphism $\tau$ extends to a morphism $\sigma: Q T \rightarrow Q S$ in $\mathcal{F}$ satisfying $\left.\sigma\right|_{Q}=\operatorname{Id}_{Q}$. Thus replacing $S$ by $T$ and $\psi$ by $\psi \circ \sigma$ concludes the proof.

Corollary 6.3. Let $A$ be an almost source algebra of a block with defect group $P$ and fusion system $\mathcal{F}$. Let $Q, R$ be subgroups of $P$ such that $Q$ is fully $\mathcal{F}$-centralised. If $\operatorname{Hom}_{\mathcal{F}}(Q, R)=\emptyset$, then $(A \underset{\mathcal{O} R}{\otimes} \mathcal{O})(Q)=\{0\}$.

Corollary 6.4. Let $A, B$ be almost source algebras of blocks of finite groups having a common defect group $P$ and the same fusion system $\mathcal{F}$. Let $Q, R$ be subgroups of $P$ such that $Q$ is fully $\mathcal{F}$-centralised. If $\operatorname{Hom}_{\mathcal{F}}(Q, R)=\emptyset$, then $(A \otimes B)(\Delta Q)=\{0\}$.

Proof. This follows from Corollary 6.3 in conjunction with Propositions 4.9 and 2.2 .

Corollary 6.5. Let $A$ be an almost source algebra of a block with defect group $P$ and fusion system $\mathcal{F}$. Let $Q$ be a fully $\mathcal{F}$-centralised subgroup of $P$. Every indecomposable direct summand of $(A \underset{\mathcal{O Q}}{\otimes} \mathcal{O})(Q)$ is isomorphic to $A(\Delta Q) m_{k Z(Q)}^{\otimes} k$ for some primitive idempotent $m \in A(\Delta Q)$.

Proof. By Theorem 6.2, an indecomposable direct summand $Y$ of $(A \otimes \mathcal{O})(Q)$ is isomorphic to a direct summand of $A(\Delta Q) \underset{k S}{\otimes} \underset{k}{ }$ for some subgroup $S$ of $C_{P}(Q)$ containing $Z(Q)$ and some primitive idempotent $m \in A(\Delta Q)^{\Delta S}$ satisfying $\operatorname{Br}_{\Delta S}(m) \neq$ 0 such that $Q S$ is isomorphic to a subgroup of $Q$ in $\mathcal{F}$. This forces $S \subseteq Q$, hence $S=Z(Q)$. Since the image of $Z(Q)$ in $A(\Delta Q)^{\times}$belongs to $Z(A(\Delta Q))$, a primitive idempotent in $A(\Delta Q)^{Z(Q)}$ is primitive in $A(\Delta Q)$, and hence the module $A(\Delta Q) m_{k Z(Q)}^{\otimes} k$ is indecomposable since it is an indecomposable projective module for the algebra $A(\Delta Q) \underset{k Z(Q)}{\otimes} k$. 


\section{GROTHENDIECK GROUPS OF TRIVIAL AND LINEAR SOURCE MODULES FOR ALMOST SOURCE ALGEBRAS}

In this section we translate some of the concepts and terminology related to trivial source and linear source modules for block algebras to the source algebra level.

Definition 7.1. Let $A, B$ be $\mathcal{O}$-algebras. We denote by $\mathcal{R}(A, B)$ the Grothendieck group of $A$ - $B$-bimodules which are finitely generated as left $A$-modules and as right $B$-modules, with respect to split exact sequences. We denote by $\mathcal{P}(A, B)$ the subgroup of $\mathcal{R}(A, B)$ generated by the $A$-B-bimodules which are finitely generated projective as left $A$-modules and as right $B$-modules. For $A, B, C$ three $\mathcal{O}$-algebras we denote by

$$
-_{B}-: \mathcal{R}(A, B) \times \mathcal{R}(B, C) \longrightarrow \mathcal{R}(A, C)
$$

the group homomorphism induced by the tensorproduct $M \underset{B}{\otimes} N$ over $B$ of an $A$ - $B$ bimodule $M$ and a $B$-C-bimodule $N$. We consider $\mathcal{R}(A)=\mathcal{R}(A, A)$ as a unitary associative ring with multiplication - - and a unit element $[A]$, the isomorphism class of $A$ as an $A$ - $A$-bimodule in $\mathcal{R}(A)$, and we consider $\mathcal{R}(A, B)$ as an $\mathcal{R}(A)$ $\mathcal{R}(B)$-bimodule via $-\dot{A}_{A}-$ and $-\dot{B}_{B}-$. Clearly the bilinear map $-\dot{B}_{B}-$ induces a bilinear map

$$
-{ }_{B}-: \mathcal{P}(A, B) \times \mathcal{P}(B, C) \longrightarrow \mathcal{P}(A, C) ;
$$

in particular, $\mathcal{P}(A)=\mathcal{P}(A, A)$ is a unitary subring of $\mathcal{R}(A)$.

Following [21, given a finite group $P$, an interior $P$-algebra is an $\mathcal{O}$-algebra $A$ endowed with a group homomorphism $P \rightarrow A^{\times}$, or equivalently, endowed with a unitary algebra homomorphism $\mathcal{O P} \rightarrow A$.

Definition 7.2. Let $P$ be a finite group and let $A, B$ be interior $P$-algebras. We denote by $\mathcal{T}(A, B)$ the subgroup of $\mathcal{R}(A, B)$ generated by the finite direct sums of summands of the $A$-B-bimodules $A \otimes B Q B$, where $Q$ runs over the subgroups of $P$.

We will also need a source algebra version of linear source modules. Let $Q$ be a finite $p$-group. Any group homomorphism $\theta: Q \rightarrow \mathcal{O}^{\times}$induces an $\mathcal{O}$-algebra automorphism sending $u \in Q$ to $\theta(u) u$. Given an $\mathcal{O} Q$-module $U$ we denote by ${ }_{\theta} U$ the $\mathcal{O} Q$-module which is equal to $U$ as an $\mathcal{O}$-module, with $u \in Q$ acting as $\theta(u) u$ on $U$. We use a similar notation for right modules and bimodules.

Definition 7.3. Let $P$ be a finite $p$-group and let $A, B$ be interior $P$-algebras. We denote by $\mathcal{L}(A, B)$ the Grothendieck group of finite direct sums of summands of $A$-B-bimodules of the form $A \underset{\mathcal{O} Q}{\otimes}{ }_{\theta} B$, with $Q$ running over the subgroups of $P$ and $\theta$ over the set $\operatorname{Hom}\left(Q, \mathcal{O}^{\times}\right)$of group homomorphisms from $Q$ to $\mathcal{O}^{\times}$.

Clearly $\mathcal{T}(A, B)$ is a subgroup of $\mathcal{L}(A, B)$. If $A, B$ are finitely generated projective over $\mathcal{O} P$, then $\mathcal{T}(A, B)$ and $\mathcal{L}(A, B)$ are subgroups of $\mathcal{P}(A, B)$. If $A$ is isomorphic to a direct summand of $A \underset{\mathcal{O} P}{\otimes} A$ as an $A$-A-bimodule, then $[A]$ belongs to $\mathcal{T}(A)=\mathcal{T}(A, A)$. By Proposition 4.2 this case occurs whenever $A$ is an almost source algebra of a block with $P$ as its defect group. The groups $\mathcal{T}(A, B)$ and $\mathcal{L}(A, B)$ are independent of the choice of almost source idempotents in the following sense. 
Theorem 7.4. Let $G, H$ be finite groups and let b, c be blocks of $\mathcal{O} G, \mathcal{O} H$ with a common defect group $P$, respectively. Let $i \in(\mathcal{O} G b)^{\Delta P}, j \in(\mathcal{O H} H)^{\Delta P}$ be almost source idempotents. Set $A=i \mathcal{O} G i$ and $B=j \mathcal{O} H j$. Let $i^{\prime} \in(\mathcal{O} G b)^{\Delta P}, j^{\prime} \in$ $(\mathcal{O H} c)^{\Delta P}$ be idempotents satisfying $i=i i^{\prime}=i^{\prime} i$ and $j=j j^{\prime}=j^{\prime} j$. Set $A^{\prime}=i^{\prime} \mathcal{O} G i^{\prime}$ and $B^{\prime}=j^{\prime} \mathcal{O} H j^{\prime}$. The map sending an $A$-B-bimodule $M$ to the $A^{\prime}$-B'-bimodule $A^{\prime} i \underset{A}{\otimes} M \underset{B}{\otimes} j B^{\prime}$ induces injective group homomorphisms

$$
\begin{aligned}
& \mathcal{T}(A, B) \longrightarrow \mathcal{T}\left(A^{\prime}, B^{\prime}\right), \\
& \mathcal{L}(A, B) \longrightarrow \mathcal{L}\left(A^{\prime}, B^{\prime}\right) .
\end{aligned}
$$

If the idempotents $i^{\prime}, j^{\prime}$ are also almost source idempotents and if $i, j$ determine the same fusion system on $P$, then these maps are isomorphisms whose inverses are induced by the map sending an $A^{\prime}$ - $B^{\prime}$-bimodule $N$ to the $A$-B-bimodule $i N j$.

Proof. The condition $i=i i^{\prime}=i^{\prime} i$ is equivalent to $i \in A^{\prime}$, so the statement makes sense. Let $M$ be a direct summand of $A \underset{\mathcal{O} R}{\otimes}{ }_{\theta} B$ for some subgroup $R$ of $P$ and some

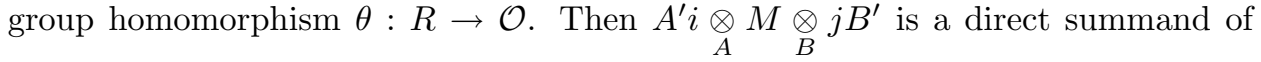
$A^{\prime} i \underset{A}{\otimes} A_{\mathcal{O} R}^{\otimes}{ }_{\theta} B \underset{B}{\otimes} j B^{\prime} \cong A^{\prime} i \underset{\mathcal{O} R}{\otimes}{ }_{\theta} j B^{\prime}$, which is a direct summand of $A^{\prime} \underset{\mathcal{O} R}{\otimes}{ }_{\theta} B^{\prime}$, Thus the map sending $M$ to $A^{\prime} i \otimes \otimes_{A} M \underset{B}{\otimes} j B^{\prime}$ induces group homomorphisms $\mathcal{T}(A, B) \rightarrow$ $\mathcal{T}\left(A^{\prime}, B^{\prime}\right)$ and $\mathcal{L}(A, B) \rightarrow \mathcal{L}\left(A^{\prime}, B^{\prime}\right)$. These group homomorphisms are injective because $A, A^{\prime}$ and $B, B^{\prime}$ are Morita equivalent via the bimodules $A^{\prime} i, B^{\prime} j$ and their duals, respectively, as a consequence of Proposition 4.1. Suppose now that $i^{\prime}$ and $j^{\prime}$ are almost source idempotents. In order to show the surjectivity of the above maps we may assume that $i, j$ are source idempotents. Let $N$ be an indecomposable direct summand of $A^{\prime} \underset{\mathcal{O} R}{\otimes}{ }_{\theta} B^{\prime}$ for some group homomorphism $\theta: Q \rightarrow \mathcal{O}^{\times}$. Thus $N$ is an indecomposable direct summand of $A^{\prime} n \underset{\mathcal{O} R}{\otimes} \theta s B^{\prime}$ for some primitive idempotents $n \in\left(A^{\prime}\right)^{\Delta R}$ and $s \in\left(B^{\prime}\right)^{\Delta R}$. By choosing $R$ of minimal order we may assume that $\operatorname{Br}_{\Delta R}(n) \neq 0$ and $\operatorname{Br}_{\Delta R}(s) \neq 0$. In other words, $n$ belongs to a local point $\nu$ of $Q$ on $A^{\prime}$, and $s$ belongs to a local point $\sigma$ of $R$ on $B^{\prime}$. Since $i^{\prime}, j^{\prime}$ are almost source idempotents, the defect group $P$ has unique local points $\gamma, \delta$ on $A^{\prime}, B^{\prime}$, respectively, and so $i \in \gamma$ and $j \in \delta$. Denote by $\mathcal{F}$ the common fusion system of $G$ and $H$ on $P$. Let $\varphi: Q \cong R$ be an isomorphism in $\mathcal{F}$ such that $Q$ is fully $\mathcal{F}$-centralised. By Proposition 5.1 there is a primitive idempotent $m \in A^{\Delta Q}$ satisfying $\operatorname{Br}_{\Delta Q}(m) \neq 0$ such that $\mathcal{O} G m \cong(\mathcal{O} G n)_{\varphi}$ as $\mathcal{O} G b$ - $\mathcal{O} Q$-bimodules. Multiplying by $i^{\prime}$ yields an isomorphism $A^{\prime} m \cong\left(A^{\prime} n\right)_{\varphi}$ as $A^{\prime}$-OOQ $Q$-bimodules. Similarly, there is a primitive idempotent $r \in B^{\Delta Q}$ satisfying $\operatorname{Br}_{\Delta Q}(r) \neq 0$ such that $r B^{\prime} \cong{ }_{\varphi} s B^{\prime}$ as $\mathcal{O} Q-B^{\prime}$ bimodules. Then ${ }_{\theta \circ \varphi} r B^{\prime} \cong{ }_{\varphi \theta} s B^{\prime}$ as $\mathcal{O} Q$ - $B^{\prime}$-bimodules. Thus $N$ is isomorphic to a direct summand of $A^{\prime} n \underset{\mathcal{O} R}{\otimes} \theta^{\theta} s B^{\prime} \cong A^{\prime} m \underset{\mathcal{O} Q}{\otimes} \tau s B^{\prime}$, where $\tau=\theta \circ \varphi: Q \rightarrow \mathcal{O}^{\times}$. Since $m \in A, r \in B$ the latter is isomorphic to a direct summand of $A^{\prime} i{ }_{\mathcal{O} Q}^{\otimes} \tau j B^{\prime}$. Thus $N$ is in fact a direct summand of $A^{\prime} i \underset{\mathcal{O Q}}{\otimes} \tau j B^{\prime}$. Multiplication by $i$ on the left and by $j$ on the right is a Morita equivalence between the category of $A^{\prime}-B^{\prime}$ bimodules and the category of $A$-B-bimodules. Thus, setting $M=i N j$ we get that

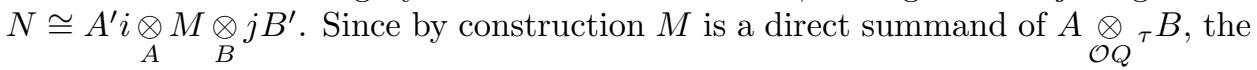
surjectivity of the given maps follows. 
Following the notation in [4, 1.2], if $G, H$ are finite groups, $b$ a block of $\mathcal{O} G$ and $c$ a block of $\mathcal{O} H$ with a common defect group $P$, then $T^{\Delta P}(\mathcal{O} G b, \mathcal{O} H c)$ denotes the Grothendieck group of $\mathcal{O} G b-\mathcal{O} H c$-bimodules (with respect to split exact sequences) whose indecomposable direct summands have trivial source and a vertex contained in $\Delta P$, when viewed as $\mathcal{O}(G \times H)$-modules. Similarly, $L^{\Delta P}(\mathcal{O} G b, \mathcal{O} H c)$ denotes the Grothendieck group of linear source $\mathcal{O} G b-\mathcal{O} H c$-bimodules (with respect to split exact sequences) whose indecomposable direct summands have a linear source and a vertex contained in $\Delta P$. The following consequence of Theorem 7.4 implies in particular that the notion of $p$-permutation equivalence as introduced by Boltje and $\mathrm{Xu}$ is equivalent to the source algebra version of Definition 1.3 above in case the involved blocks are of principal type. Recall that $b$ is said to be of principal type if $\operatorname{Br}_{\Delta Q}(b)$ is a block of $k C_{G}(Q)$ for every subgroup $Q$ of a defect group $P$ of $b$. Equivalently, $b$ is of principal type if and only if $b$ itself is an almost source idempotent. If $b$ is the principal block of $\mathcal{O} G$, then by Brauer's Third Main Theorem, $\operatorname{Br}_{\Delta Q}(b)$ is the principal block of $k C_{G}(Q)$ for any subgroup $Q$ of a Sylow- $p$-subgroup $P$ of $G$, and hence in particular and not surprisingly, the principal block $b$ is of principal type.

Corollary 7.5. Let $G, H$ be finite groups and let $b, c$ be blocks of $\mathcal{O} G, \mathcal{O} H$ with a common defect group $P$, respectively. Let $i \in(\mathcal{O} G b)^{\Delta P}, j \in(\mathcal{O H} c)^{\Delta P}$ be almost source idempotents. Set $A=i \mathcal{O} G i$ and $B=j \mathcal{O} H j$. The map sending an $A-B$ bimodule $M$ to the $\mathcal{O} G b-\mathcal{O} H$ c-bimodule $\mathcal{O} G i \underset{A}{\otimes}{\underset{B}{\otimes}}_{\underset{0}{\otimes}}^{\otimes} \mathcal{O} H$ induces injective group homomorphisms

$$
\begin{aligned}
& \mathcal{T}(A, B) \longrightarrow T^{\Delta P}(\mathcal{O} G b, \mathcal{O} H c), \\
& \mathcal{L}(A, B) \longrightarrow L^{\Delta P}(\mathcal{O} G b, \mathcal{O} H c) .
\end{aligned}
$$

If the blocks b, $c$ are of principal type and have the same fusion system, these maps are isomorphisms.

Proof. For any subgroup $R$ of $P$ and any group homomorphism $\theta: R \rightarrow \mathcal{O}^{\times}$we have an isomorphism of $\mathcal{O}(G \times H)$-modules

$$
\mathcal{O} G \underset{\mathcal{O} R}{\otimes}{ }_{\theta} \mathcal{O} H \cong \operatorname{Ind}_{\Delta R}^{G \times H}\left({ }_{\theta} \mathcal{O}\right)
$$

sending $x \otimes y^{-1}$ to $(x, y) \otimes 1$ for $x \in G, y \in H$. Thus $\mathcal{T}(\mathcal{O} G b, \mathcal{O} H c) \cong T^{\Delta P}(\mathcal{O} G b, \mathcal{O} H c)$ and $\mathcal{L}(\mathcal{O} G b, \mathcal{O} H c) \cong L^{\Delta P}(\mathcal{O} G b, \mathcal{O} H c)$. Thus Theorem 7.4 applied to $i^{\prime}=b$ and $j^{\prime}=c$ yields the injective group homomorphisms as stated. If $b$ and $c$ are of principal type, then $b$ and $c$ are almost source idempotents, and hence, if they determine the same fusion system, it follows again from Theorem 7.4 that the maps in the statement are isomorphisms.

Remark 7.6. For non-principal blocks the maps in Corollary 7.5 need not be isomorphisms, in general. If $\mathcal{O} G b$ is a block algebra whose defect group $P$ has two different local points $\gamma, \gamma^{\prime}$ on $\mathcal{O} G b$, then $\mathcal{O} G i \underset{\mathcal{O P}}{\otimes} i^{\prime} \mathcal{O} G$ is a $p$-permutation $\mathcal{O}(G \times G)$-module, where $i \in \gamma, i^{\prime} \in \gamma^{\prime}$, but not all indecomposable summands of $i \mathcal{O} G i \otimes i^{\prime} \mathcal{O} G i$ as $i \mathcal{O} G i$-iO Gi-bimodules will be summands of bimodules of the form $i \mathcal{O} G i \underset{\mathcal{O} Q}{\mathcal{O} P} i \mathcal{O} G i$. This is because $\gamma^{\prime}={ }^{x} \gamma$ for some $x \in N_{G}(P)$ not contained in $N_{G}\left(P_{\gamma}\right)$, so conjugation by $x$ is an automorphism of $P$ which does not belong to the $A$-fusion on $P$. 
For the sake of completeness we include some further observations relating the notation of the present paper to that in 44. Let $A$ be an almost source algebra of a block $b$ of a finite group $G$ with defect group $P$. Consider $\mathcal{O}$ as a trivial interior $P$-algebra with structural homomorphism $P \rightarrow \mathcal{O}^{\times}$sending any element $u \in P$ to $1_{\mathcal{O}}$. Then $\mathcal{T}(A, \mathcal{O})$ is the Grothendieck group of sums of summands of the left $A$-modules $A \underset{\mathcal{O} Q}{\otimes}$, where $Q$ runs over the subgroups of $P$. Similarly, $\mathcal{L}(A, \mathcal{O})$ is the Grothendieck group of sums of summands of the left $A$-modules $A \otimes{ }_{0} \mathcal{O}$, where $Q \subseteq P$ and $\theta \in \operatorname{Hom}\left(Q, \mathcal{O}^{\times}\right)$. Following the notation of Boltje and $\mathrm{Xu}$ [4, we denote by $T(\mathcal{O} G b)$ the Grothendieck group of $p$-permutation $\mathcal{O} G b$-modules; that is, $T(\mathcal{O} G b)$ is generated by the isomorphism classes of summands of $\mathcal{O} G b \underset{\mathcal{O Q}}{\otimes} \mathcal{O}$, with $Q \subseteq P$. Similarly, we denote by $L(\mathcal{O} G b)$ the Grothendieck group generated by the linear source $\mathcal{O} G b$-modules; that is, $L(\mathcal{O} G b)$ is generated by the isomorphism classes of summands of $\mathcal{O} G b \underset{\mathcal{O} Q}{\otimes}{ }_{\theta} \mathcal{O}$, with $Q \subseteq P$ and $\theta \in \operatorname{Hom}\left(Q, \mathcal{O}^{\times}\right)$.

Proposition 7.7. Let $G$ be a finite group, let $b$ be a block of $\mathcal{O} G$, let $P$ be a defect group of b, let $i \in(\mathcal{O} G b)^{\Delta P}$ be an idempotent satisfying $\operatorname{Br}_{\Delta P}(i) \neq 0$ and set $A=i \mathcal{O} G i$. The functor $\mathcal{O} G i \otimes-: \bmod (A) \rightarrow \bmod (\mathcal{O} G b)$ induces group isomorphisms $\mathcal{T}(A, \mathcal{O}) \cong T(\mathcal{O} G b)$ and $\mathcal{L}(A, \mathcal{O}) \cong L(\mathcal{O} G b)$.

Proof. Let $Q$ be a subgroup of $P$, let $\theta: Q \rightarrow \mathcal{O}^{\times}$be a group homomorphism and let $Y$ be a direct summand of $A \underset{\mathcal{O Q}}{\otimes}{ }_{\theta} \mathcal{O}$. Then $\mathcal{O} G i \underset{A}{\otimes} Y$ is a direct summand of $\mathcal{O} G i \underset{\mathcal{O Q}}{\otimes}$ ${ }_{\theta} \mathcal{O}$, hence of $\mathcal{O} G b \underset{\mathcal{O} Q}{\otimes}{ }_{\theta} \mathcal{O}$. Thus the functor $\mathcal{O} G i \otimes_{A}-$ induces group homomorphisms $\mathcal{T}(A, \mathcal{O}) \rightarrow T(\mathcal{O} G b)$ and $\mathcal{L}(A, \mathcal{O}) \rightarrow L(\mathcal{O} G b)$. These group homomorphisms are injective because the functor $\mathcal{O} G i \otimes-$ is an equivalence, by Proposition 4.1. To see that they are also surjective, let $X$ be an indecomposable linear source $\mathcal{O} G$ module. It follows from [13, 6.3] that one can choose a vertex $R$ of $X$ such that $X$ is isomorphic to a direct summand of $\mathcal{O} G i \underset{\mathcal{O} R}{\otimes} \rho \mathcal{O}$ for some group homomorphism $\rho: R \rightarrow \mathcal{O}^{\times}$. Thus the functor $\mathcal{O} G i \underset{A}{\otimes}-$ induces the isomorphisms as stated.

With the notation of Corollary 7.5, the map given by $-\dot{\mathcal{O} H c}-$ sends $T^{\Delta P}(\mathcal{O} G b, \mathcal{O} H c) \times L(\mathcal{O} H c)$ to $L(\mathcal{O} G b)$ (cf. [2, 2.3]). Clearly the map - ${ }_{B}-$ sends $\mathcal{T}(A, B) \times \mathcal{L}(B, \mathcal{O})$ to $\mathcal{L}(A, \mathcal{O})$. These two maps correspond to each other via the maps from Corollary 7.5 and Proposition 7.7:

Proposition 7.8. Let $G, H$ be finite groups and let $b, c$ be blocks of $\mathcal{O} G, \mathcal{O} H$ with a common defect group $P$, respectively. Let $i \in(\mathcal{O} G b)^{\Delta P}, j \in(\mathcal{O} H c)^{\Delta P}$ be almost source idempotents. Set $A=i \mathcal{O} G i$ and $B=j \mathcal{O} H j$. We have a commutative diagram

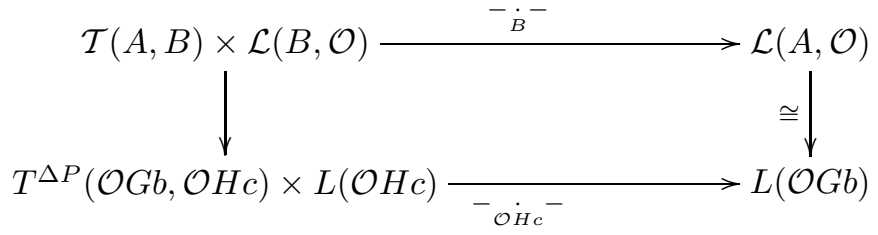

where the vertical arrows are induced by those in Corollary 7.5 and Proposition 7.7. 
Proof. Let $M$ be a direct summand of an $A$-B-bimodule of the form $A \underset{\mathcal{O} Q}{\otimes} B$ for some subgroup $Q$ of $P$ and let $Y$ be a direct summand of a left $B$-module $B \underset{\mathcal{O} R}{\otimes}{ }_{\theta} \mathcal{O}$ for some subgroup $R$ of $P$ and some group homomorphism $\theta: R \rightarrow \mathcal{O}^{\times}$. Then $M \otimes Y$ is a direct summand of $A \underset{\mathcal{O} Q}{\otimes} B \underset{\mathcal{O} R}{\otimes}{ }_{\theta} \mathcal{O}$. Since $B$ is a permutation $\mathcal{O}(Q \times R)$-module it follows that $M \underset{B}{\otimes} Y$ is a direct sum of linear source $A$-modules. The composition of maps $\mathcal{T}(A, B) \times \mathcal{L}(B, \mathcal{O}) \rightarrow T^{\Delta P}(\mathcal{O} G b, \mathcal{O} H c) \times L(\mathcal{O} H c) \rightarrow L(\mathcal{O} G b)$ is induced by the map

$$
\begin{aligned}
(M, Y) & \mapsto(\mathcal{O} G i \underset{A}{\otimes} M \underset{B}{\otimes} j \mathcal{O} H, \mathcal{O} H j \underset{B}{\otimes} Y) \mapsto \mathcal{O} G i \underset{A}{\otimes} M \underset{B}{\otimes} j \mathcal{O} H \underset{\mathcal{O} H c}{\otimes} \mathcal{O} H j \underset{B}{\otimes} Y \\
& \cong \mathcal{O} G i \underset{A}{\otimes} M{\underset{B}{\otimes}}^{\otimes} Y,
\end{aligned}
$$

and the composition of maps $\mathcal{T}(A, B) \times \mathcal{L}(B, \mathcal{O}) \rightarrow \mathcal{L}(B, \mathcal{O}) \cong L(\mathcal{O} G b)$ is induced by the map

$$
(M, Y) \mapsto M \underset{B}{\otimes} Y \mapsto \mathcal{O} G i \underset{A}{\otimes} M \underset{B}{\otimes} Y
$$

hence both maps coincide.

\section{Ring And module Structures of $\mathcal{T}(A, B)$ And $\mathcal{L}(A, B)$}

As a consequence of [17, §2] we get some extra structure on $\mathcal{T}(A, B)$ in case $A$, $B$ are source algebras. The following theorem extends [17, 2.3.(i)] to linear source modules over almost source algebras.

Theorem 8.1. Let $A, B, C$ be almost source algebras of p-blocks of finite groups with a common defect group $P$ all having the same fusion system $\mathcal{F}$ on $P$. The tensorproduct over $B$ induces bilinear maps

$$
\begin{gathered}
-{ }_{B}-: \mathcal{L}(A, B) \times \mathcal{L}(B, C) \longrightarrow \mathcal{L}(A, C), \\
-{ }_{B}-: \mathcal{T}(A, B) \times \mathcal{T}(B, C) \longrightarrow \mathcal{T}(A, C) .
\end{gathered}
$$

Proof. Let $M$ be an indecomposable direct summand of $A \underset{\mathcal{O} Q}{\otimes}{ }_{\theta} B$ and let $N$ be an indecomposable direct summand of $B \underset{\mathcal{O} R}{\otimes}{ }_{\eta} C$ for some subgroups $Q, R$ of $P$ and some group homomorphisms $\theta: Q \rightarrow \mathcal{O}^{\times}$and $\eta: R \rightarrow \mathcal{O}^{\times}$. Let $X$ be an indecomposable direct summand of $M \underset{B}{\otimes} N$. We have to show that $X$ is isomorphic to a direct summand of $A \underset{\mathcal{O} S}{\otimes}{ }_{\tau} C$ for some subgroup $S$ of $P$ and some group homomorphism $\tau: S \rightarrow \mathcal{O}^{\times}$, such that $\tau$ is trivial whenever both $\theta, \eta$ are trivial. The bimodule $X$ is isomorphic to a direct summand of

$$
A \underset{\mathcal{O} Q}{\otimes}{ }_{\theta} B \underset{\mathcal{O} R}{\otimes}{ }_{\eta} C
$$

Since $X$ is indecomposable, there is an indecomposable direct summand $W$ of $B$ as an $\mathcal{O} Q-\mathcal{O} R$-bimodule such that $X$ is isomorphic to a direct summand of

$$
A \underset{\mathcal{O} Q}{\otimes}{ }_{\theta} W \underset{\mathcal{O} R}{\otimes}{ }_{\eta} C
$$

By Proposition 5.2(i), the $\mathcal{O} Q-\mathcal{O} R$-bimodule $W$ is isomorphic to $\mathcal{O} Q \underset{\mathcal{O} S}{\otimes} \varphi \mathcal{O}_{R}$ for some subgroup $S$ of $Q$ and some morphism $\varphi: S \rightarrow R$ belonging to the fusion 
system $\mathcal{F}$. Thus $X$ is isomorphic to a direct summand of

$$
A \underset{\mathcal{O} S}{\otimes} \varphi\left({ }_{\sigma} C\right)
$$

where $\sigma: \varphi(S) \rightarrow \mathcal{O}^{\times}$is the group homomorphism sending $\varphi(y)$ to $\theta(y) \eta(\varphi(y))$, for every $y \in S$. Since $X$ is indecomposable, $X$ is in fact isomorphic to a direct summand of $A n \underset{\mathcal{O} S}{\otimes} \varphi\left({ }_{\sigma} s C\right)$ for some primitive idempotent $n \in A^{\Delta S}$ and some primitive idempotent $s \in C^{\Delta \varphi(S)}$. By [15, 2.6] we may assume that $n, s$ belong to local points of $S, \varphi(S)$ on $A, C$, respectively. Let $T$ be a fully $\mathcal{F}$-centralised subgroup of $P$ such that there is an isomorphism $\psi: T \rightarrow S$ in $\mathcal{F}$. By Proposition 5.1 there are primitive idempotents $m \in A^{\Delta T}$ and $r \in C^{\Delta T}$ such that $A m \cong A n_{\psi}$ as $A$-OT-bimodules, and such that $r C \cong{ }_{\varphi \psi} s C$ as $\mathcal{O} T$ - $C$-bimodules. Thus $X$ is a direct summand of

$$
A m \underset{\mathcal{O} T}{\otimes} \tau r C
$$

where $\tau=\sigma \circ \psi: T \rightarrow \mathcal{O}^{\times}$. This shows that the isomorphism class of $X$ belongs to $\mathcal{L}(A, C)$. By construction, if $\theta, \eta$ are trivial, so is $\tau$, and hence in that case, the isomorphism class of $X$ belongs to $\mathcal{T}(A, C)$.

Corollary 8.2. Let $A$ be an almost source algebra of a p-block of a finite group with defect group $P$. The abelian groups $\mathcal{T}(A)$ and $\mathcal{L}(A)$ are unitary subrings of $\mathcal{P}(A)$.

Proof. Applying Theorem 8.1 to $A=B=C$ shows that $\mathcal{T}(A)$ and $\mathcal{L}(A)$ are rings; the fact that they are unitary follows from Proposition 4.2.

Corollary 8.3. Let $A, B$ be almost source algebras of p-blocks of finite groups with a common defect group $P$ and the same fusion system. Then $\mathcal{L}(A, B)$ is an $\mathcal{L}(A)$ $\mathcal{L}(B)$-bimodule and $\mathcal{T}(A, B)$ is an $\mathcal{T}(A)-\mathcal{T}(B)$-bimodule, with left and right module structure induced by the tensorproducts over $A$ and $B$, respectively.

Proof. This follows from Theorem 8.1 applied to the cases $A=B$ and $B=C$.

Corollary 8.4. Let $A, B$ be almost source algebras of p-blocks of finite groups with a common defect group $P$ and the same fusion system. Let $X$ be a p-permutation equivalence between $A$ and $B$. The map sending $Y \in \mathcal{T}(A)$ to $X^{*}{ }_{A} Y{ }_{A} X$ is a ring isomorphism

$$
\mathcal{T}(A) \cong \mathcal{T}(B)
$$

Proof. For $Y, Z$ in $\mathcal{T}(A)$ we have $\left(X^{*} \cdot Y \cdot X\right) \cdot\left(X_{A}^{*} \cdot{ }_{A} \cdot X\right)=X_{A}^{*}{ }_{A} Y \dot{A}_{A}\left(X \cdot_{B} X^{*}\right) \cdot Z$ $\dot{A}_{A} X=X_{A}^{*} \dot{A}_{A} \cdot[A] \cdot Z{ }_{A} X=X_{A}^{*} \cdot(Y \cdot Z) \cdot X$, which shows that the given map is a ring homomorphism. Exchanging the roles of $A, B$ and $X, X^{*}$ yields the inverse.

The ring structure of $\mathcal{T}(A)$ does not depend on the choice of the almost source idempotents:

Proposition 8.5. Let $G$ be a finite group, let $b$ be a block of $\mathcal{O} G$, let $P, P^{\prime}$ be defect groups of $b$, and let $i \in(\mathcal{O} G b)^{\Delta P}$ and $i^{\prime} \in(\mathcal{O} G b)^{\Delta P^{\prime}}$ be almost source idempotents. Set $A=i \mathcal{O} G i$ and $A^{\prime}=i^{\prime} \mathcal{O} G i^{\prime}$. There is a canonical ring isomorphism $\mathcal{T}(A) \cong$ $\mathcal{T}\left(A^{\prime}\right)$. 
Proof. As a consequence of Theorem 7.4 we may assume that $i, i^{\prime}$ are source idempotents. Let $\gamma, \gamma^{\prime}$ be the local points of $P, P^{\prime}$ on $\mathcal{O} G b$ containing $i, i^{\prime}$, respectively. By [21, 1.2] there is $x \in G$ such that $P_{\gamma^{\prime}}^{\prime}={ }^{x}\left(P_{\gamma}\right)$. We may assume that $i^{\prime}={ }^{x} i$. Let $\alpha: A \cong A^{\prime}$ be the $\mathcal{O}$-algebra isomorphism sending $a \in A$ to ${ }^{x} a \in A^{\prime}$. Then $\alpha$ induces an isomorphism of $A$-A-bimodules

$$
A \underset{\mathcal{O} Q}{\otimes} A \cong{ }_{\alpha} A^{\prime} \underset{\mathcal{O} \alpha(Q)}{\otimes} A_{\alpha}^{\prime}
$$

where we have identified $Q$ to its image in $A$. Thus the map sending an $A-A$ bimodule $M$ to the $A^{\prime}$ - $A^{\prime}$-bimodule $\alpha_{\alpha^{-1}} M_{\alpha^{-1}}$ induces a ring isomorphism $\mathcal{T}(A) \cong$ $\mathcal{T}\left(A^{\prime}\right)$. We need to show that this isomorphism does not depend on the choice of $x$. If $y$ is another element such that $P_{\gamma^{\prime}}^{\prime}={ }^{y}\left(P_{\gamma}\right)$, then $y=x n$ for some $n \in N_{G}\left(P_{\gamma}\right)$, so it suffices to show that the automorphism of $\mathcal{T}(A)$ induced by $n$ is the identity. By [22, 2.12] there is an element $c \in A^{\times}$which normalises the image of $P$ in $A$ such that conjugation by $c$ induces the same group automorphism $P$ as conjugation by $n$. Denote by $\beta$ the inner automorphism of $A$ sending $a \in A$ to ${ }^{c} a$. Then $\beta$ induces for any $A$-A-bimodule $M$ an isomorphism of $A$-A-bimodules $M \cong{ }_{\beta} M_{\beta}$. The result follows.

Given two $\mathcal{O}$-algebras $A, B$, the $\mathcal{O}$-dual $M^{*}=\operatorname{Hom}_{\mathcal{O}}(M, \mathcal{O})$ of an $A$ - $B$-bimodule is a $B$-A-bimodule via $(b . \mu . a)(m)=\mu(a m b)$ for $a \in A, b \in B, m \in M$ and $\mu \in M^{*}$. Thus, taking $\mathcal{O}$-duality induces a group homomorphism $\mathcal{R}(A, B) \cong \mathcal{R}(B, A)$. Recall that an $\mathcal{O}$-algebra $A$ is called symmmetric if $A$ is free of finite rank as an $\mathcal{O}$-module and if $A \cong A^{*}$ as an $A$ - $A$-bimodule. Group algebras of finite groups, their block algebras and almost source algebras are symmetric.

Proposition 8.6. Let $A, B$ be symmetric $\mathcal{O}$-algebras. Taking $\mathcal{O}$-duals of bimodules induces a group isomorphism $\mathcal{P}(A, B) \cong \mathcal{P}(B, A)$ and an anti-automorphism on the $\operatorname{ring} \mathcal{P}(A)$.

Proof. Since $A, B$ are symmetric, the $\mathcal{O}$-dual of an $A$ - $B$-bimodule which is finitely generated projective as a left $A$-module and as a right $B$-module is again finitely generated projective as a left $B$-module and as a right $A$-module. This shows the first statememt. If $M, N$ are $A$ - $A$-bimodules which are finitely generated projective as left and right $A$-modules, then $(M \underset{A}{\otimes} N)^{*} \cong N^{*} \underset{A}{\otimes} M^{*}$ which implies the second statement.

Proposition 8.7. Let $A, B$ be almost source algebras of blocks of finite groups with a common defect group $P$. Taking $\mathcal{O}$-duals of bimodules induces group isomorphisms $\mathcal{L}(A, B) \cong \mathcal{L}(B, A)$ and $\mathcal{T}(A, B) \cong \mathcal{T}(B, A)$, as well as anti-automorphisms of the rings $\mathcal{L}(A)$ and $\mathcal{T}(A)$.

Proof. Since $A, B, \mathcal{O} P$ are symmetric, the $\mathcal{O}$-dual of an $A$ - $B$-bimodule of the form $A \underset{\mathcal{O} Q}{\otimes}{ }_{\theta} B$ for some subgroup $Q$ of $P$ and a group homomorphism $\theta: Q \rightarrow \mathcal{O}^{\times}$ is isomorphic to the $B$ - $A$-bimodule $B_{\theta} \underset{\mathcal{O} Q}{\otimes} A \cong B \underset{\mathcal{O} Q}{\otimes}\left(\theta^{-1}\right) A$. The first statement follows. The second statement follows from Proposition 8.6.

Given a finite group $G$, any $p$-permutation $k G$-module lifts uniquely, up to isomorphism, to a $p$-permutation $\mathcal{O} G$-module. Conversely, a linear source $\mathcal{O} G$-module reduces modulo $J(\mathcal{O})$ to a trivial source $k G$-module because there are no non-trivial 
group homomorphisms from a finite $p$-group to $k^{\times}$. At the source algebra level, this translates to the following statement:

Proposition 8.8. Let $A, B$ be almost source algebras of blocks with a common defect group $P$. The map sending an $A$-B-bimodule $M$ to $k \underset{\mathcal{O}}{\otimes} M$ induces a surjective group homomorphism $\mathcal{L}(A, B) \rightarrow \mathcal{T}(k \underset{\mathcal{O}}{\otimes} A, k \underset{\mathcal{O}}{\otimes} B)$ and a group isomorphism $\mathcal{T}(A, B) \cong \mathcal{T}(k \underset{\mathcal{O}}{\otimes} A, k \underset{\mathcal{O}}{\otimes} B)$. For $A=B$ this is a ring isomorphism $\mathcal{T}(A) \cong \mathcal{T}\left(k \otimes_{\mathcal{O}} A\right)$.

Proof. If $A$ and $B$ are almost source algebras of blocks $b$ and $c$ of finite groups $G$ and $H$, respectively, with a common defect group $P$, then $A \underset{\mathcal{O}}{\otimes}$ is an almost source algebra of the block $b \otimes c$ of $G \times H$. Via the Morita equivalence between a block algebra and its almost source algebra any direct summand of $\bar{A} \underset{k Q}{\otimes} \bar{B}$ corresponds to a $\Delta P$-projective $p$-permutation $k(G \times H)$-module, and hence lifts uniquely, up to isomorphism, to a direct summand of $A \otimes B$, where $Q$ is a subgroup of $P$ and where $\bar{A}=k \underset{\mathcal{O}}{\otimes} A, \bar{B}=k \underset{\mathcal{O}}{\otimes} B$. The result follows.

\section{Compatibility with the Brauer construction}

The first result in this section says that under suitable hypotheses, the Brauer construction sends $\mathcal{T}(A, B)$ to $\mathcal{T}(A(\Delta Q), B(\Delta Q))$. For $P$ abelian this is [4, 3.3].

Theorem 9.1. Let $A, B$ be almost source algebras of p-blocks b, $c$ of finite groups $G, H$, respectively, with a common defect group $P$ having the same fusion system $\mathcal{F}$ on $P$. Let $Q$ be a fully $\mathcal{F}$-centralised subgroup of $P$. For any $X \in \mathcal{T}(A, B)$ we have $X(\Delta Q) \in \mathcal{T}(A(\Delta Q), B(\Delta Q))$.

Proof. Let $i \in(\mathcal{O} G b)^{\Delta P}$ and $j \in(\mathcal{O H})^{\Delta P}$ be almost source idempotents such that $A=i \mathcal{O} G i$ and $B=j \mathcal{O} H j$. Denote by $c^{0}$ and $j^{0}$ the images in $\mathcal{O} H$ of $c$ and $j$, respectively, of the anti-automorphism of $\mathcal{O} H$ sending $y \in H$ to $y^{-1}$. Then $B^{o p} \cong j^{0} \mathcal{O} H j^{0}$ is an almost source algebra of the block $c^{0}$ of $\mathcal{O} H$, by Proposition 4.10. Let $R$ be a subgroup of $P$. We need to show that $(A \underset{\mathcal{O} R}{\otimes} B)(\Delta Q)$ is a direct sum of summands of the $A(\Delta Q)-B(\Delta Q)$-bimodules $A(\Delta Q) \underset{k S}{\otimes} B(\Delta Q)$, with $S$ running over the subgroups of $C_{P}(Q)$. Let $Y$ be an indecomposable direct summand of $(A \underset{\mathcal{O} R}{\otimes} B)(\Delta Q)$. As $A \underset{\mathcal{O}}{\otimes} B^{o p}$-modules we have an isomorphism

$$
A \underset{\mathcal{O} R}{\otimes} B \cong\left(A \underset{\mathcal{O}}{\otimes} B^{o p}\right) \underset{\mathcal{O} \Delta R}{\otimes} \mathcal{O}
$$

sending $x \otimes y$ to $(x \otimes y) \otimes 1_{\mathcal{O}}$, for $x \in A$ and $y \in B$. By Proposition 4.9, the algebra $A \otimes B^{o p}$ is an almost source algebra of the block $b \otimes c^{0}$ of $G \times H$. By the assumptions, $A, B$ and hence $B^{o p}$ determine the same fusion system $\mathcal{F}$ on $P$ (cf. Proposition 4.10). Since $Q$ is fully $\mathcal{F}$-centralised, $\Delta Q$ is fully $\mathcal{F} \times \mathcal{F}$-centralised by Proposition 2.2. We now apply Theorem 6.2 to $A \underset{\mathcal{O}}{\otimes} B^{o p}, P \times P, \mathcal{F} \times \mathcal{F}, \Delta Q, \Delta R$ instead of $A$, $P, \mathcal{F} Q, R$, respectively. The conclusion of Theorem 6.2 yields a fully $C_{\mathcal{F} \times \mathcal{F}}(\Delta Q)$ centralised subgroup $S$ of $C_{P}(Q) \times C_{P}(Q)$ and a morphism $\psi: \Delta Q \cdot S \rightarrow \Delta R$ in $\mathcal{F} \times \mathcal{F}$ such that $Y$ is isomorphic to a direct summand of $\left(A \underset{\mathcal{O}}{\otimes} B^{o p}\right)(\Delta Q) \underset{k S}{\otimes} k$ as 
$\left(A \underset{\mathcal{O}}{\otimes} B^{o p}\right)(\Delta Q)$-modules. Note that we have obvious isomorphisms

$$
\left(A \underset{\mathcal{O}}{\otimes} B^{o p}\right)(\Delta Q) \cong A(\Delta Q) \underset{k}{\otimes} B^{o p}(\Delta Q) \cong A(\Delta Q) \underset{k}{\otimes} B(\Delta Q)^{o p}
$$

Since $\psi: \Delta Q \cdot S \rightarrow \Delta R$ is an injective group homomorphism, the group $S$ must be of the form

$$
S=\{(t, \tau(t)) \mid t \in T\}
$$

for some subgroup $T$ of $C_{P}(Q)$ and some injective group homomorphism $\tau: T \rightarrow$ $C_{P}(Q)$. Since $\psi$ is a morphism in $\mathcal{F} \times \mathcal{F}$ there are morphisms $\psi_{1}: Q T \rightarrow P$ and $\psi_{2}: Q \tau(T) \rightarrow P$ such that

$$
\psi(u t, v \tau(s))=\left(\psi_{1}(u t), \psi_{2}(v \tau(t))\right)
$$

for all $u, v \in Q$ and $t, s \in T$. Since $\psi(\Delta Q) \subseteq \Delta R$ we have $\psi_{1}(u)=\psi_{2}(u)$ for all $u \in Q$. Also, since $\psi(S) \subseteq \Delta R$ we have $\psi_{1}(t)=\psi_{2}(\tau(t))$ for all $t \in T$. Thus $\left.\psi_{2}^{-1} \circ \psi_{1}\right|_{Q}=\operatorname{Id}_{Q}$ and $\left.\psi_{2}^{-1} \circ \psi_{1}\right|_{T}=\tau$. This proves that $\tau$ is a morphism in $C_{\mathcal{F}}(Q)$. Thus we have an isomorphism

$$
\Delta(Q T) \cong \Delta Q \cdot S
$$

in $\mathcal{F} \times \mathcal{F}$ mapping $(u t, u t)$ to $(u, u) \cdot(t, \tau(t))$ for all $u \in Q$ and $t \in T$. Now $S$ is fully $C_{\mathcal{F} \times \mathcal{F}}(\Delta Q)$-centralised and $C_{\mathcal{F} \times \mathcal{F}}(\Delta Q)=C_{\mathcal{F}}(Q) \times C_{\mathcal{F}}(Q)$ by Proposition 2.3. Thus, by Proposition 2.2, in particular $T$ is fully $C_{\mathcal{F}}(Q)$-centralised. But then, by Proposition 2.2 again, $\Delta T$ is fully $C_{\mathcal{F}}(Q) \times C_{\mathcal{F}}(Q)$-centralised. Thus, by combining Proposition 5.1 and Theorem 6.2 we may replace $S$ by $\Delta T$. This, however, shows that $Y$ is isomorphic to a direct summand of

$$
\left(A(\Delta Q) \underset{k}{\otimes} B(\Delta Q)^{o p}\right) \underset{k \Delta T}{\otimes} k \cong A(\Delta Q) \underset{k T}{\otimes} B(\Delta Q) .
$$

Thus the isomorphism class of $Y$ belongs to $\mathcal{T}(A(\Delta Q), B(\Delta Q))$. The result follows.

The second theorem in this section extends similar statements in [15, 2.4], 17, 2.3.(ii)], and [4, 3.6], and includes in particular a proof of Theorem 1.2.

Theorem 9.2. Let $A, B$ be almost source algebras of $p$-blocks of finite groups with a common defect group $P$ having the same fusion system $\mathcal{F}$ on $P$ and let $C$ be an interior $P$-algebra. Let $Q$ be a fully $\mathcal{F}$-centralised subgroup of $P$ and let $\theta: Q \rightarrow \mathcal{O}^{\times}$ be a group homomorphism. For any $X \in \mathcal{T}(A, B)$ and any $Y \in \mathcal{R}(B, C)$ we have

$$
\left(X \dot{B}_{B} Y\right)(\Delta Q, \Delta \theta)=X(\Delta Q) \underset{B(\Delta Q)}{.} Y(\Delta Q, \Delta \theta),
$$

where $\Delta \theta: \Delta Q \rightarrow \mathcal{O}^{\times}$is the group homomorphism sending $(u, u)$ to $\theta(u)$ for all $u \in Q$. In particular, for any fully $\mathcal{F}$-centralised subgroup $Q$ of $P$ the map sending $X \in \mathcal{T}(A)$ to $X(\Delta Q)$ is a ring homomorphism $\rho_{Q}: \mathcal{T}(A) \rightarrow \mathcal{T}(A(\Delta Q))$.

Proof. Let $M$ be an $A$ - $B$-bimodule and let $N$ be a $B$ - $C$-bimodule. Consider the obvious maps

$$
M^{\Delta Q} \underset{\mathcal{O}}{\otimes} N^{(\Delta Q, \Delta \theta)} \rightarrow(M \underset{\mathcal{O}}{\otimes} N)^{(\Delta Q, \Delta \theta)} \rightarrow(M \underset{B}{\otimes} N)^{(\Delta Q, \Delta \theta)} \rightarrow(M \underset{B}{\otimes} N)(\Delta Q, \Delta \theta) .
$$

The composition of these maps induces a homomorphism of $A(\Delta Q)-C(\Delta Q)$-bimodules

$$
M(\Delta Q) \underset{B(\Delta Q)}{\otimes} N(\Delta Q, \Delta \theta) \longrightarrow(M \underset{B}{\otimes} N)(\Delta Q, \Delta \theta) .
$$


If we fix $N$ and let $M$ vary, this map is an additive functorial in $M$. Thus, in order to show that this is an isomorphism if $M$ is a finite direct sum of summands of bimodules of the form $A \underset{\mathcal{O} R}{\otimes} B$, we may assume that $M$ is a direct summand of $A \underset{\mathcal{O} R}{\otimes} B$ as an $\mathcal{O} Q$ - $B$-bimodule for some subgroup $R$ of $P$. It suffices to show that this map is a $k$-linear isomorphism, and so we may ignore the left $A(\Delta Q)$-module structure. Thus we may in fact assume that $M=W \underset{\mathcal{O} R}{\otimes} B$ for some indecomposable direct summand $W$ of $A$ as an $\mathcal{O} Q-\mathcal{O} R$-bimodule, for some subgroup $R$ of $P$. By Proposition 5.2 we have $W \cong \mathcal{O} Q \underset{\mathcal{O} T}{\otimes} \varphi \mathcal{O} R$ for some subgroup $T$ of $Q$ and some group homomorphism $\varphi: T \rightarrow R$ belonging to the common fusion system of $A$ and $B$ on $P$. If $T$ is a proper subgroup of $Q$, then both sides in the above map are zero. Assume that $Q=T$, hence $M \cong{ }_{\varphi} B$ as an $\mathcal{O} Q$ - $B$-bimodule. By decomposing $M$ further we may in fact assume that $M={ }_{\varphi} n B$ for some primitive idempotent $n \in B^{\Delta \varphi(Q)}$. Then again, if $\operatorname{Br}_{\Delta \varphi(Q)}(n)=0$, both sides in the above map are easily seen to be zero. Thus we may assume that $\operatorname{Br}_{\Delta \varphi(Q)}(n) \neq 0$. As $Q$ is fully $\mathcal{F}$-centralised, we can apply Proposition 5.1 , which shows that there is a primitive idempotent $m \in B^{\Delta Q}$ such that ${ }_{\varphi} n B \cong m B$ as an $\mathcal{O} Q$ - $B$-bimodules. Thus we may assume $M=m B$. But then $M$ is a direct summand of $B$ itself, so we may assume that $M=B$ as $\mathcal{O} Q-B$-bimodule. But in that case, both sides in the above map are canonically isomorphic to $N(\Delta Q)$. The last statement follows by applying this to $A=B=C$ and $\theta=1$.

If one translates Theorem 9.2 back to modules over block algebras, one gets in particular the following statement (needed in the proof of Theorem 1.4):

Corollary 9.3. Let $G, H$ be finite groups, let $b$ be a block of $\mathcal{O} G$, and let $c$ be a block of $\mathcal{O H}$. Suppose that $b$ and $c$ have a common defect group $P$ and that there are source idempotents $i \in(\mathcal{O} G b)^{\Delta P}$ and $j \in(\mathcal{O} H c)^{\Delta P}$ such that the fusion systems on $P$ determined by $i$ and $j$ coincide. For any subgroup $Q$ of $P$ denote by $e_{Q}$ the unique block of $k C_{G}(Q)$ satisfying $\operatorname{Br}_{\Delta Q}(i) e_{Q} \neq 0$ and by $f_{Q}$ the unique block of $k C_{H}(Q)$ satisfying $\operatorname{Br}_{\Delta Q}(j) f_{Q} \neq 0$. Let $M$ be an $\mathcal{O} G b-\mathcal{O H c}$-bimodule which is a finite direct sum of summands of the bimodules $\mathcal{O} G i \otimes j \mathcal{O} H$, with $Q$ running over the subgroups of $P$. For any $\mathcal{O} H c$-module $U$, any subgroup $Q$ of $P$ and any group homomorphism $\theta: Q \rightarrow \mathcal{O}^{\times}$we have an isomorphism of $k C_{G}(Q) e_{Q}$-modules

$$
e_{Q}(M(\Delta Q)) f_{Q} \underset{k C_{H}(Q) f_{Q}}{\otimes} f_{Q}(U(Q, \theta)) \cong e_{Q}((M \underset{k H c}{\otimes} U)(Q, \theta)) .
$$

Proof. By conjugating $Q$ simultaneously we may assume that $Q$ is fully centralised with respect to the common fusion system of $A=i \mathcal{O} G i$ and $B=j \mathcal{O} H j$. Then, by making use of the Morita equivalences between $A(\Delta Q), k C_{G}\left(e_{Q}\right)$ and between $B(\Delta Q), k C_{H}(Q) f_{Q}$, from Proposition 4.6 it suffices to show that there is an isomorphism of $A(\Delta Q)$-modules

$$
\operatorname{Br}_{\Delta Q}(i) M(\Delta Q) \operatorname{Br}_{\Delta Q}(j) \underset{B(\Delta Q)}{\otimes} \operatorname{Br}_{\Delta Q}(j) U(Q, \theta) \cong \operatorname{Br}_{\Delta Q}(i)((M j \underset{B}{\otimes} j U)(Q, \theta)) .
$$

Now the left side is isomorphic to $(i M j)(\Delta Q) \underset{B(\Delta Q)}{\otimes}(j U)(Q, \theta)$ by Lemma 3.9, and the right side is isomorphic to $(i M j \underset{B}{\otimes} j U)(Q, \theta)$. Both are isomorphic thanks to Theorem 9.2 applied to $A, B, C=\mathcal{O}$, the isomorphism class of $i M j$ instead of $X$ and the isomorphism class of $j U$ instead of $Y$. 
Remark 9.4. Theorem 9.1 adds to the results in [15, 17. that if $X$ is a splendid tiliting complex of $A$-B-bimodules, then for any $\mathcal{F}$-centric subgroup $Q$ of $P$ the Rickard complex $X(\Delta Q)$ of $A(\Delta Q)-B(\Delta Q)$-bimodules is splendid (in the slightly more restrictive sense of the definition given in [15, 1.10] or [17, 1.1]).

Remark 9.5. With the notation of Theorem 9.2 , if $Q$ is a fully $\mathcal{F}$-centralised subgroup of $P$ and $S$ a fully $C_{\mathcal{F}}(Q)$-centralised subgroup of $C_{P}(Q)$, then $R=Q S$ is fully $\mathcal{F}$-centralised by Proposition 2.1. By the usual transitivity properties of the Brauer construction we have $A(\Delta Q)(\Delta S)=A(\Delta R)$, and the following diagram of ring homomorphisms is commutative:

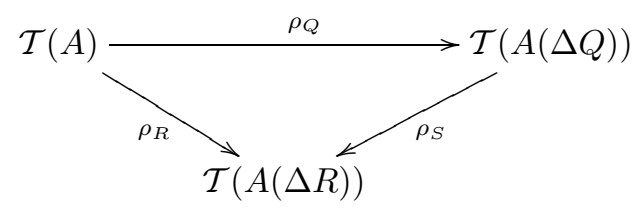

\section{ISOTYPIES}

Let $G, H$ be finite groups, let $b$ be a block of $\mathcal{O} G$ and let $c$ be a block of $\mathcal{O} H$. Suppose that the quotient field $K$ of $\mathcal{O}$ is large enough for $b, c$ and their Brauer pairs. As before, denote by $\mathbb{Z} \operatorname{Irr}_{K}(G, b)$ the group of generalised characters of $G$ over $K$ associated with the block $b$, and denote by $\mathbb{Z I B r}_{K}(G, b)$ the corresponding group of generalised Brauer characters. Following Broué, a perfect isometry between $b$ and $c$ is a group isomorphism

$$
\Psi: \mathbb{Z} \operatorname{Irr}_{K}(H, c) \cong \mathbb{Z} \operatorname{Irr}_{K}(G, b)
$$

sending $\chi \in \operatorname{Irr}_{K}(H, c)$ to $\epsilon(\chi) \eta_{\chi}$ for some $\epsilon(\chi) \in\{1,-1\}$ and some $\eta_{\chi} \in \operatorname{Irr}_{K}(G, b)$ such that the character $\mu \in \mathbb{Z} \operatorname{Irr}(H \times G)$ defined by

$$
\mu(y, x)=\sum_{\chi \in \operatorname{Irr}_{K}(H, c)} \epsilon(\chi) \chi(y) \eta_{\chi}\left(x^{-1}\right)
$$

for all $(y, x) \in H \times G$ has the following properties: the character value $\mu(y, x)$ is divisible in $\mathcal{O}$ by the orders of $C_{G}(x), C_{H}(y)$, for all $x \in G$, all $y \in H$, and $\mu(y, x)=0$ if exactly one of $x, y$ is a $p^{\prime}$-element. Since the decomposition map $\mathbb{Z I r r}_{K}(G, b) \rightarrow \mathbb{Z I B r}_{K}(G, b)$, given by restricting a generalised character to the set of $p^{\prime}$-elements in $G$, is surjective, the arithmetic properties of a perfect isometry $\Psi$ imply that $\Psi$ induces an isomorphism

$$
\bar{\Psi}: \mathbb{Z I B r}_{K}(H, c) \cong \mathbb{Z} \operatorname{IBr}_{K}(G, b)
$$

Since $\mu$ determines the map $\Psi$ by the formula $\Psi(\chi)(x)=\frac{1}{|H|} \sum_{y \in H} \mu(y, x) \chi\left(y^{-1}\right)$ for all $x \in G$ and all $\chi \in \mathbb{Z}_{\operatorname{Irr}_{K}}(H, c)$, we will sometimes abusively use the same notation for $\Psi$ and $\mu$.

Suppose now that $b$ and $c$ have a common defect group $P$, let $i \in(\mathcal{O} G b)^{\Delta P}$ and $j \in(\mathcal{O H})^{\Delta P}$ be source idempotents. Suppose that the fusion systems of the source algebras $i \mathcal{O} G i$ and $j \mathcal{O} H j$ on $P$ are equal. For any subgroup $Q$ of $P$ denote by $e_{Q}$ the unique block of $k C_{G}(Q)$ satisfying $\operatorname{Br}_{\Delta Q}(i) e_{Q} \neq 0$ and by $f_{Q}$ the unique block of $k C_{H}(Q)$ satisfying $\operatorname{Br}_{\Delta Q}(j) f_{Q} \neq 0$. Denote by $\hat{e}_{Q}$ and $\hat{f}_{Q}$ the blocks of 
$\mathcal{O} C_{G}(Q)$ and $\mathcal{O} C_{H}(Q)$ which lift $e_{Q}$ and $f_{Q}$, respectively. Again following Broué, an isotypy between $b$ and $c$ is a family of perfect isometries

$$
\Psi_{Q}: \mathbb{Z}_{\operatorname{Irr}_{K}}\left(C_{H}(Q), \hat{f}_{Q}\right) \cong \mathbb{Z}_{\operatorname{Irr}_{K}}\left(C_{G}(Q), \hat{e}_{Q}\right)
$$

for every subgroup $Q$ of $P$, with the following properties:

(1) for any isomorphism $\varphi: Q \cong R$ in the common fusion system $\mathcal{F}$ we have ${ }^{\varphi} \Psi_{Q}=\Psi_{R}$, where ${ }^{\varphi} \Psi_{Q}$ is obtained by composing $\Psi_{Q}$ with the isomorphisms $\mathbb{Z} \operatorname{Irr}_{K}\left(C_{G}(Q), \hat{e}_{Q}\right) \cong \mathbb{Z}_{\operatorname{Irr}_{K}}\left(C_{G}(R), \hat{e}_{R}\right)$ and $\mathbb{Z} \operatorname{Irr}_{K}\left(C_{H}(Q), \hat{f}_{Q}\right) \cong \mathbb{Z I r r}_{K}\left(C_{H}(R), \hat{f}_{R}\right)$, given by conjugation with elements $x \in G, y \in H$ satisfying $\varphi(u)=x u x^{-1}=y u y^{-1}$ for all $u \in Q$;

(2) for any subgroup $Q$ of $P$, any element $u \in C_{P}(Q)$, setting $R=Q\langle u\rangle$, we have an equality of maps

$$
d_{\left(C_{G}(Q), e_{Q}\right)}^{\left(u, e_{R}\right)} \circ \Psi_{Q}=\bar{\Psi}_{R} \circ d_{\left(C_{H}(Q), f_{Q}\right)}^{\left(u, e_{R}\right)}
$$

from $\mathbb{Z} \operatorname{Irr}_{K}\left(C_{H}(Q), \hat{f}_{Q}\right)$ to $K \underset{\mathbb{Z}}{\otimes} \mathbb{Z} \operatorname{IBr}_{K}\left(C_{G}(R), \hat{e}_{R}\right)$, where $d_{\left(C_{G}(Q), e_{Q}\right)}^{\left(u, e_{R}\right)}$ is the generalised decomposition map from $\mathbb{Z I r r}_{K}\left(C_{G}(Q), \hat{e}_{Q}\right)$ to $K \underset{\mathbb{Z}}{\otimes} \mathbb{Z} \operatorname{IBr}_{K}\left(C_{G}(R), \hat{e}_{R}\right)$ as mentioned in $\S 3.7$ and $d_{\left(C_{H}(Q), f_{Q}\right)}^{\left(u, f_{R}\right)}$ is defined analogously.

Theorem 10.1. Let $G, H$ be finite groups, let $b$ be a block of $\mathcal{O} G$, and let $c$ be $a$ block of $\mathcal{O} H$. Suppose that $b$ and $c$ have a common defect group $P$ and that there are source idempotents $i \in(\mathcal{O} G b)^{\Delta P}$ and $j \in(\mathcal{O H})^{\Delta P}$ such that the fusion systems on $P$ of the source algebras $A=i \mathcal{O} G i$ and $B=j \mathcal{O} H j$ coincide. Suppose there is a $p$-permutation equivalence $X$ between $A$ and $B$. Then for any subgroup $Q$ of $P$ the character $\Psi_{Q}$ of $C_{G}(Q) \times C_{H}(Q)$ determined by $e_{Q}(\mathcal{O} G i \otimes X \otimes j \mathcal{O} H)(\Delta Q) f_{Q}$ is a perfect isometry between the unique blocks $e_{Q}, f_{Q}$ of $C_{G}(\stackrel{A}{Q}), C_{H}^{B}(Q)$ determined by $i, j$, respectively, and the family $\left\{\Psi_{Q} \mid Q \subseteq P\right\}$ is an isotypy between $b$ and $c$.

Proof. Observe the abuse of notation in the following statement. We mean by $e_{Q}((\mathcal{O} G i \underset{A}{\otimes} X \underset{B}{\otimes} j \mathcal{O} H)(\Delta Q)) f_{Q}$ the image in $\left.T\left(k C_{G}(Q) e_{Q}\right), k C_{H}(Q) f_{Q}\right)$ of $X$ under the map which is induced by the map sending an $A$-B-bimodule $M$ to the $k C_{G}(Q) e_{Q}-k C_{H}(Q) f_{Q}$-bimodule $e_{Q}((\mathcal{O} G i \otimes M \underset{B}{\otimes} j \mathcal{O} H)(\Delta Q)) f_{Q}$. This could have been denoted more accurately by $e_{Q}\left(\left([O G i]{ }_{A} X_{B} \cdot[j \mathcal{O} H]\right)(\Delta Q)\right) f_{Q}$. Condition (1) holds clearly. Thus, in order to show that $\Psi_{Q}$ induces a perfect isometry, we may assume that $Q$ is fully $\mathcal{F}$-centralised. Set

$$
Y_{Q}=e_{Q}((\mathcal{O} G i \underset{A}{\otimes} X \underset{B}{\otimes} j \mathcal{O} H)(\Delta Q)) f_{Q} .
$$

By Proposition 4.11 we have

$$
Y_{Q}=k C_{G}(Q) \operatorname{Br}_{\Delta Q}(i) \underset{A(\Delta Q)}{\otimes} X(\Delta Q) \underset{B(\Delta Q)}{\otimes} \operatorname{Br}_{\Delta Q}(j) k C_{H}(Q) .
$$

By Theorem 9.1, $X(\Delta Q)$ belongs to $\mathcal{T}(A(\Delta Q), B(\Delta Q)$ and hence, by Corollary 7.5, $Y_{Q}$ belongs to $T^{\Delta C_{P}(Q)}\left(k C_{G}(Q) e_{Q}, k C_{H}(Q) f_{Q}\right)$. Since $p$-permutation modules over $k$ lift uniquely, up to isomorphism, to $p$-permutation modules over $\mathcal{O}$ preserving vertices, $Y_{Q}$ lifts to a unique element $\hat{Y}_{Q}$ in $T^{\Delta C_{P}(Q)}\left(\mathcal{O} C_{G}(Q) \hat{e}_{Q}, \mathcal{O} C_{H}(Q) \hat{f}_{Q}\right)$. Moreover, $Y_{Q}$ is a virtual module which is projective on the left and on the right. Therefore, in order to show that this induces a perfect isometry it suffices to show 
that it induces an isometry. The fact that $p$-permutation modules lift uniquely as mentioned above implies that it suffices to show that

$$
Y_{Q} \underset{k C_{H}(Q) f_{Q}}{ } Y_{Q}^{*}=\left[k C_{G}(Q) e_{Q}\right]
$$

Since $Q$ is fully $\mathcal{F}$-centralised, cutting with $\operatorname{Br}_{\Delta Q}(i)$ and $\operatorname{Br}_{\Delta Q}(j)$ yields Morita equivalences. Thus, it suffices to show that

$$
\operatorname{Br}_{\Delta Q}(i) Y_{Q} \operatorname{Br}_{\Delta Q}(i) \underset{B(\Delta Q)}{.} \operatorname{Br}_{\Delta Q}(i) Y_{Q}^{*} \operatorname{Br}_{\Delta Q}(i)=[A(\Delta Q)] .
$$

Using Proposition 4.11 we get

$$
\operatorname{Br}_{\Delta Q}(i) Y_{Q} \operatorname{Br}_{\Delta Q}(i)=X(\Delta Q),
$$

and hence this is simply equivalent to showing that

$$
X(\Delta Q) \underset{B(\dot{\Delta} Q)}{\dot{2}} X(\Delta Q)^{*}=[A(\Delta Q)] .
$$

Thanks to Theorem 9.2 we have

$$
X(\Delta Q) \underset{B(\Delta Q)}{\dot{D}_{(1)}} X(\Delta Q)^{*}=\left(X \dot{B}^{*}\right)(\Delta Q)=[A](\Delta Q)=[A(\Delta Q)]
$$

as required, and thus $\Psi_{Q}$ is a perfect isometry. The commutativity (2) follows from translating the argument given by Boltje and $\mathrm{Xu}$ in [4,3.8(e)], with $\operatorname{Br}_{P}(e), \operatorname{Br}_{Q}(e)$, $\operatorname{Br}_{P}(f), \operatorname{Br}_{Q}(f)$ replaced by $e_{Q}, e_{R}, f_{Q}, f_{R}$, respectively, where the notation is now as in (2). Note that $f_{R}$ is a block of $C_{C_{G}(Q)}(u)=C_{G}(R)$. In what follows we write $d^{\left(u, e_{R}\right)}$ instead of $d_{\left(C_{G}(Q), \hat{e}_{Q}\right)}^{\left(u, e_{R}\right)}$, and smilarly we write $d^{\left(u, f_{R}\right)}$ instead of $d_{\left(C_{H}(Q), \hat{f}_{Q}\right)}^{\left(u, f_{R}\right)}$ We need to show the equality

$$
\left(d^{\left(u, e_{R}\right)} \circ \Psi_{Q}\right)(\chi)=\left(\bar{\Psi}_{R} \circ d^{\left(u, f_{R}\right)}\right)(\chi)
$$

for any $\chi \in \mathbb{Z}_{\operatorname{Irr}_{K}}\left(C_{H}(Q), \hat{f}_{Q}\right)$. By a theorem of Brauer, every generalised character is a $\mathbb{Z}$-linear combination of characters of linear source modules. Thus it suffices to prove the above equality in the case where $\chi$ is the character of a linear source $\mathcal{O} C_{H}(Q) \hat{f}_{Q}$-module $U$. Then $\Psi_{Q}(\chi)$ is the character of $\hat{Y}_{Q} \otimes U$ (which in line with our convention on abuse of notation means $\hat{Y}_{Q} \underset{\mathcal{O} C_{H}(Q) \hat{f}_{Q}}{ }\left[\begin{array}{l}\mathcal{O} C_{H}(Q) \hat{f}_{Q} \\ {[}\end{array}\right.$ again a virtual linear source $\mathcal{O} C_{G}(Q) \hat{e}_{Q}$-module (cf. Proposition 7.8). Thus, by Theorem 3.8, we get that

$$
d^{\left(u, e_{R}\right)}\left(\Psi_{Q}(\chi)\right)=\sum_{\theta \in \operatorname{Hom}(\langle u\rangle, \mathcal{O} \times)} \theta(u) \otimes\left[\left[f_{R}\left(\left(\hat{Y}_{Q} \underset{\mathcal{O C}_{H}(Q) \hat{f}_{Q}}{\otimes} U\right)(\langle u\rangle, \theta)\right)\right]\right] .
$$

Similarly, again by Theorem 3.8 , we have

$$
d^{\left(u, f_{R}\right)}(\chi)=\sum_{\theta \in \operatorname{Hom}(\langle u\rangle, \mathcal{O} \times)} \theta(u) \otimes\left[\left[f_{R}(U(\langle u\rangle, \theta))\right]\right] .
$$

Applying $\bar{\Psi}_{R}$ to this expression yields

$$
\bar{\Psi}_{R}\left(d^{\left(u, f_{R}\right)}(\chi)\right)=\sum_{\theta \in \operatorname{Hom}\left(\langle u\rangle, \mathcal{O}^{\times}\right)} \theta(u) \otimes\left[\left[Y_{R} \underset{k C_{H}(R) f_{R}}{\otimes} f_{R}(U(\langle u\rangle, \theta))\right]\right] .
$$

Since $Y_{R}=e_{R}\left(\hat{Y}_{Q}(\Delta\langle u\rangle)\right) f_{R}$, the required equality of these two expressions now follows from Corollary 9.3. 


\section{InVARIANCE UNDER MORITA EQUIVALENCES WITH ENDO-PERMUTATION SOURCE}

If $b, c$ are blocks of finite groups $G, H$ such that the block algebras $\mathcal{O} G b, \mathcal{O} H c$ are Morita equivalent via an $\mathcal{O} G b-\mathcal{O} H c$-bimodule $M$ with endo-permutation source $V$, then by a result of Puig [24], $b$ and $c$ have isomorphic defect groups, and there is an identification of defect groups of $b$ and $c$ such that for some choice of source idempotents the corresponding fusion systems are equal and such that $V$ is stable with respect to this fusion system (see e.g. [12] for the terminology regarding fusion stable endo-permutation modules). Thus Theorem 1.6 is a consequence of the following theorem:

Theorem 11.1. Let $A, B$ be source algebras of blocks b, c of finite groups $G$, $H$ with a common defect group $P$ and the same fusion system $\mathcal{F}$ on $P$. Suppose that there is an indecomposable $\mathcal{F}$-stable endo-permutation $\mathcal{O} P$-module $V$ and an indecomposable direct summand $M$ of the $A$-B-bimodule $A \underset{\mathcal{O} P}{\otimes} \operatorname{Ind}_{\Delta P}^{P \times P}(V) \underset{\mathcal{O} P}{\otimes} B$ satisfying $M \otimes M^{*} \cong A$ and $M^{*} \otimes M \cong B$. Then the map sending an $A$-A-bimodule

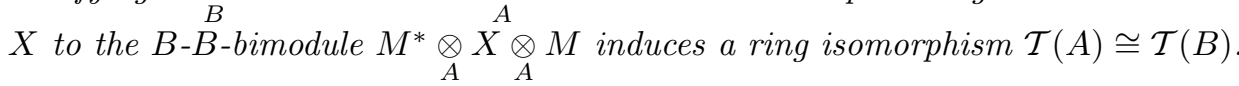

Proof. We need to show that for $Q$ a subgroup of $P$, the isomorphism class of the $B$ - $B$-bimodule $M^{*} \otimes A \otimes A \otimes M$ belongs to $\mathcal{T}(B)$. By the assumptions on $M$, the bimodule $M^{*} \otimes A \otimes A \otimes M$ is isomorphic to a direct summand of $A \quad \mathcal{O} \quad A$

$B \otimes \operatorname{Ind}_{\Delta P}^{P \times P}\left(V^{*}\right) \otimes A \otimes A \otimes \operatorname{Ind}_{\Delta P}^{P \times P}(V) \otimes B$. It follows from Proposition 5.2 $\mathcal{O} P$ O $P$ O $P Q \mathcal{O} P$ indecomposable direct summand of $A \otimes A$ as an $\mathcal{O} P$ - $\mathcal{O} P$-bimodule is that every indecomposable direct summand of $A \underset{\mathcal{O} Q}{\otimes} A$ as an $\mathcal{O} P$-O $\mathcal{O}$-bimodule is isomorphic to $\mathcal{O} P \underset{\mathcal{O} R}{\otimes} \varphi \mathcal{O} P$ for some subgroup $R$ of $P$ and some $\varphi \in \operatorname{Hom}_{\mathcal{F}}(R, P)$. Thus, if $Y$ is an indecomposable direct summand of the bimodule $M^{*} \underset{A}{\otimes} A_{\mathcal{O} Q}^{\otimes} A \otimes_{A} M$, then $Y$ is isomorphic to a direct summand of $B \underset{\mathcal{O} P}{\otimes} \operatorname{Ind}_{\Delta P}^{P \times P}\left(V^{*}\right) \underset{\mathcal{O} R}{\otimes} \operatorname{Ind}_{\Delta P}^{P \times P}(V) \underset{\mathcal{O} P}{\otimes} B$. One checks that

$$
\operatorname{Ind}_{\Delta P}^{P \times P}\left(V^{*}\right) \underset{\mathcal{O} R}{\otimes} \varphi \operatorname{Ind}_{\Delta P}^{P \times P}(V) \cong \operatorname{Ind}_{\Delta R}^{P \times P}\left(V^{*}{\underset{\mathcal{O}}{\otimes}}_{\varphi} V\right),
$$

and since $V$ is fusion stable, it follows that $V^{*} \otimes_{\mathcal{O}} V$ is a permutation $\mathcal{O} R$-module. Thus the isomorphism class of $Y$ belongs to $\mathcal{T}(B)$. The fact that the map sending an $A$ - $A$-bimodule $X$ to $M^{*} \otimes_{A} X \otimes_{A} M$ induces a ring homomorphism follows from the isomorphism $M \underset{B}{\otimes} M^{*} \cong B$. Finally, this is an isomorphism; its inverse is obtained by exchanging the roles of $A, B$ and of $M, M^{*}$.

Corollary 11.2. Let $A$ be a source algebra of a nilpotent block with defect group P. Then $\mathcal{T}(A) \cong \mathcal{T}(\mathcal{O} P)$.

Proof. By the main result in 23] we can identify $A=S \underset{\mathcal{O}}{\otimes} \mathcal{O} P$, where $S=\operatorname{End}_{\mathcal{O}}(V)$ for some indecomposable endo-permutation $\mathcal{O} P$-module $V$ with vertex $P$. Set

$$
M=V \underset{\mathcal{O}}{\otimes} \mathcal{O} P
$$

viewed as an $A$-O $\mathcal{O}$-bimodule via the canonical action of $S$ on $V$ and the regular actions of $\mathcal{O} P$ on the left and the right of $\mathcal{O} P$. Using the canonical isomorphism 
$S \cong V \underset{\mathcal{O}}{\otimes} V^{*}$ one shows that $M$ induces a Morita equivalence. As an $A$ - $A$-bimodule, $A$ is isomorphic to a direct summand of $A \otimes A$. Thus, as an $A$-O $P$-bimodule, $M$ is isomorphic to a direct summand of $A \underset{\mathcal{O} P}{\otimes} M=A \underset{\mathcal{O} P}{\otimes}(V \underset{\mathcal{O}}{\otimes} \mathcal{O} P)$. When considered as an $A \underset{\mathcal{O}}{\otimes} \mathcal{O} P$-module, this is isomorphic to $(A \underset{\mathcal{O}}{\otimes} \mathcal{O} P) \underset{\mathcal{O} \Delta P}{\otimes} V$ via the map sending $a \otimes(v \otimes y)$ to $\left(a \otimes y^{-1}\right) \otimes v$, for all $a \in A, v \in V$ and $y \in P$. This in turn is isomorphic to $A \underset{\mathcal{O} P}{\otimes} \operatorname{Ind}_{\Delta P}^{P \times P}(V) \underset{\mathcal{O} P}{\otimes} \mathcal{O} P$ via the map sending $\left(a \otimes y^{-1}\right) \otimes v$ to $a \otimes((1,1) \otimes v) \otimes y$, so the isomorphism $\mathcal{T}(A) \cong \mathcal{T}(\mathcal{O P})$ is a particular case of Theorem 11.1.

Remark 11.3. A nilpotent block with defect group $P$ is known to be isotypic to the defect group algebra $\mathcal{O} P$. Does any such isotypy lift to a $p$-permutation equivalence? The answer is positive whenever a source $V$ of the unique simple module has an endo-split p-permutation resolution in the sense of Rickard [25, §7], because then the isotypy is in fact induced by a splendid derived equivalence (cf. [15, 1.3] and $[25,6.3])$.

\section{Augmentation homomorphisms}

Given a finite $p$-group $P$ we denote by $B(P)$ the Burnside ring of $P$.

Proposition 12.1. Let $P$ be a finite p-group. The map sending for any subgroup $Q$ of $P$ the $P$-set $P / Q$ to the $\mathcal{O} P$-OP-bimodule $\mathcal{O} P \underset{\mathcal{O} Q}{\otimes} \mathcal{O} P$ induces a ring isomorphism

$$
\delta_{P}: B(P) \cong \mathcal{T}(\mathcal{O} P) .
$$

In particular, the ring $\mathcal{T}(\mathcal{O} P)$ is commutative and the $\mathbb{Z}$-rank of $\mathcal{T}(\mathcal{O} P)$ is equal to the number of conjugacy classes of subgroups of $P$.

Proof. One can verify this directly; alternatively, by [14, 11.4.6], the map sending an $\mathcal{O} P$-module $V$ to the $\mathcal{O} P$ - $\mathcal{O} P$-bimodule $\operatorname{Ind}_{\Delta P}^{P \times P}(V)$ induces an injective ring homomorphism from the Green ring of $\mathcal{O} P$-modules to the ring $\mathcal{P}(\mathcal{O} P)$, where here $V$ is considered as a module over $\mathcal{O} \Delta P$ via the obvious isomorphism $\Delta P \cong P$. This map sends the isomorphism class of $\mathcal{O} P / Q$ to that of $\mathcal{O} P \otimes \mathcal{O} P$, for any subgroup $Q$ of $P$, and hence induces an isomorphism $B(P) \cong \mathcal{T}(\mathcal{O} P)$ because any bimodule of the form $\mathcal{O} P \otimes \mathcal{O} O P$ is indecomposable.

The Burnside ring of a finite $p$-group $P$ admits for any subgroup $Q$ an augmentation homomorphism $\sigma_{Q}: B(P) \rightarrow \mathbb{Z}$, induced by the map sending a finite $P$-set $X$ to the number of $Q$-fixpoints $\left|X^{Q}\right|$. Composed with $\delta_{P}$ this yields an augmentation of $\mathcal{T}(\mathcal{O P})$ which can be described explicitly as follows:

Proposition 12.2. Let $P$ be a finite p-group and let $Q$ be a subgroup of $P$. The map sending an $\mathcal{O} P$-OP-bimodule $M$ to the rational number $\frac{\operatorname{dim}_{k}(M(\Delta Q)}{\left|C_{P}(Q)\right|}$ induces a ring homomorphism $\rho_{Q}: \mathcal{T}(\mathcal{O} P) \rightarrow \mathbb{Z}$, and we have $\sigma_{Q}=\rho_{Q} \circ \delta_{P}$.

Proof. Let $R$ be a subgroup of $P$. The image $P \underset{R}{\times} P$ of $P \times P$ in $\mathcal{O} P \underset{\mathcal{O} R}{\otimes} \mathcal{O} P$ is a $P$-P-stable $\mathcal{O}$-basis of $\mathcal{O} P \otimes \mathcal{O} P$. In order to calculate $(\mathcal{O} P \otimes \mathcal{O} P)(\Delta Q)$ we need to determine the set of $Q$-fixpoints in this basis. Let $y, z \in P$ and denote by $y \otimes z$ the image of $(y, z)$ in $\mathcal{O} P \underset{\mathcal{O} R}{\otimes} \mathcal{O} P$. This is a $\Delta Q$-fixpoint if and only if for every $u \in Q$ there is $r_{u} \in R$ such that $u y \otimes z=y r_{u} \otimes z=y \otimes r_{u} z=y \otimes z u$, hence if and 
only if $Q^{y} \subseteq R$ and $y z \in C_{P}(Q)$. The condition $Q^{y} \subseteq R$ is also equivalent to $y R$ being in the $Q$-fixpoint set $(P / R)^{Q}$. Note that $C_{P}(Q)$ acts freely on the left on the set of $\Delta Q$-fixpoints in the basis $P \underset{R}{\times} P$ of $\mathcal{O} P \underset{\mathcal{O} R}{\otimes} \mathcal{O} P$. Thus the map sending $y \in P$ satisfying $Q^{y} \subseteq R$ to $y \otimes y^{-1}$ induces a bijection between the $Q$-fixpoints in $P / R$ and the $C_{P}(Q)$-orbits of $\Delta Q$-fixpoints in $P \underset{R}{\times} P$. The result follows.

We get in particular an augmentation for the $\operatorname{ring} \mathcal{T}(A)$ :

Proposition 12.3. Let $A$ be a source algebra of a block of a finite group with defect group $P$. The map sending an $A$-A-bimodule $M$ to to the rational number $\frac{\operatorname{dim}_{k}(M(\Delta P))}{|Z(P)|}$ induces a unitary ring homomorphism $\alpha_{P}: \mathcal{T}(A) \rightarrow \mathbb{Z}$.

Proof. By [28, 38.10] we have $A(\Delta P) \cong k Z(P)$. Thus, by Theorem 9.2, the Brauer construction with respect to $\Delta P$ induces a unitary ring homomorphism $\mathcal{T}(A) \rightarrow$ $\mathcal{T}(k Z(P))$. The result follows from Proposition 12.2 applied to $k Z(P)$ and 1 instead of $\mathcal{O} P$ and $Q$, respectively.

As in the case of Burnside rings of finite groups, there are other homomorphisms from $\mathcal{T}(A)$ to $\mathbb{Z}$. In order to describe them, we need the following terminology.

Definition 12.4. Let $\mathcal{F}$ be a fusion system on a finite $p$-group $P$. A subgroup $Q$ of $P$ is called $\mathcal{F}$-nilpotent if $Q$ is fully $\mathcal{F}$-centralised and if $C_{\mathcal{F}}(Q)$ is the trivial fusion system $\mathcal{F}_{C_{P}(Q)}\left(C_{P}(Q)\right)$ of the group $C_{P}(Q)$ on itself.

In particular, if $Q$ is $\mathcal{F}$-centric, then $Q$ is $\mathcal{F}$-nilpotent. It is easy to check that if $Q, R$ are fully $\mathcal{F}$-centralised subgroups of $P$ such that $Q$ is $\mathcal{F}$-nilpotent and $\operatorname{Hom}_{\mathcal{F}}(Q, R) \neq \emptyset$, then $R$ is $\mathcal{F}$-nilpotent as well.

Remark 12.5. Let $G$ be a finite group, $b$ a block of $\mathcal{O} G, P$ a defect group of $b$ and $i \in(\mathcal{O} G b)^{\Delta P}$ an almost source idempotent of $b$. Set $A=i \mathcal{O} G i$, denote by $\mathcal{F}$ the fusion system of $A$ on $P$ and for any subgroup $Q$ of $P$ denote by $e_{Q}$ the unique block of $k C_{G}(Q)$ satisfying $\operatorname{Br}_{\Delta P}(i) e_{Q} \neq 0$. Then a subgroup $Q$ of $P$ is $\mathcal{F}$-nilpotent if and only if $e_{Q}$ is a nilpotent block of $k C_{G}(Q)$ having $C_{P}(Q)$ as a defect group (this is well known and follows, for instance, from [18, §2]). Therefore, if $Q$ is $\mathcal{F}$-nilpotent we have $\mathcal{T}(A(\Delta Q)) \cong \mathcal{T}\left(k C_{P}(Q)\right)$ by Corollary 11.2. Thus the composition of ring homomorphisms $\mathcal{T}(A) \rightarrow \mathcal{T}(A(\Delta Q)) \cong \mathcal{T}\left(k C_{P}(Q)\right) \rightarrow \mathbb{Z}$ yields a unitary ring homomorphism $\mathcal{T}(A) \longrightarrow \mathbb{Z}$ for any $\mathcal{F}$-nilpotent subgroup $Q$ of $P$. We can describe these ring homomorphisms explicitly:

Theorem 12.6. Let $A$ be an almost source algebra of a block with defect group $P$ and fusion system $\mathcal{F}$. Let $Q$ be an $\mathcal{F}$-nilpotent subgroup of $P$ and let $j \in A^{\Delta Q}$ be a primitive idempotent such that $\operatorname{Br}_{\Delta Q}(j) \neq 0$. The map sending an $A$-A-bimodule $M$ to the rational number $\frac{\operatorname{dim}_{k}((j M j)(\Delta Q))}{\left|C_{P}(Q)\right|}$ induces a unitary ring homomorphism $\alpha_{Q}: \mathcal{T}(A) \rightarrow \mathbb{Z}$. Moreover, $\alpha_{Q}$ is independent of the choice of $j$.

Proof. The ring homomorphism $\rho_{Q}: \mathcal{T}(A) \rightarrow \mathcal{T}(A(\Delta Q))$ is induced by the map sending an $A$-A-bimodule $M$ to $M(\Delta Q)$. The block $e_{Q}$ of $C_{G}(Q)$ determined by $A$ is nilpotent because $Q$ is $\mathcal{F}$-nilpotent. Thus $e_{Q}$ has $C_{P}(Q)$ as a defect group, there is a unique local point $\mu$ of $C_{P}(Q)$ on $A(\Delta Q)$, and for any $m \in \mu$ we have an isomorphism of the interior $C_{P}(Q)$-algebra

$$
m A(\Delta Q) m \cong S \underset{k}{\otimes} k C_{P}(Q)
$$


with $S=\operatorname{End}_{k}(V)$ for some indecomposable endo-permutation $k C_{P}(Q)$-module $V$. Every primitive idempotent in $S$ remains primitive in $m A(\Delta Q) m$ and hence lifts to a primitive idempotent $j$ in $A(\Delta Q)^{\Delta Q}$, uniquely up to conjugation, and hence

$$
(j A j)(\Delta Q) \cong k C_{P}(Q) \text {. }
$$

Thus the map sending $M$ to $(j M j)(\Delta Q)$ induces a ring homomorphism $\mathcal{T}(A) \rightarrow$ $\mathcal{T}\left(k C_{P}(Q)\right)$. Composing this with the augmentation $\mathcal{T}\left(k C_{P}(Q)\right) \rightarrow \mathbb{Z}$ obtained from Proposition 12.2 (with $C_{P}(Q)$ and 1 instead of $P$ and $Q$ ) yields the ring homomorphism $\alpha_{Q}$ as stated.

The ring homomorphisms $\alpha_{Q}$ depend only on the isomorphism class of $Q$ in the fusion system. More precisely:

Theorem 12.7. Let $A$ be a source algebra of a block of a finite group $G$ with defect group $P$ and fusion system $\mathcal{F}$. Let $Q, R$ be $\mathcal{F}$-nilpotent subgroups of $P$. We have $\alpha_{Q}=\alpha_{R}$ if and only if $Q \cong R$ in $\mathcal{F}$.

Proof. Suppose that $\alpha_{Q}=\alpha_{R}$. Let $j \in A^{\Delta Q}$ be a primitive idempotent satisfying $\operatorname{Br}_{\Delta Q}(j) \neq 0$. Then $(j A \underset{\mathcal{O} Q}{\otimes} A j)(\Delta Q)$ is non-zero, hence the image of $[A \underset{\mathcal{O} Q}{\otimes} A]$ under $\alpha_{Q}$ is non-zero. But then its image under $\alpha_{R}$ is non-zero, which forces $(A \underset{\mathcal{O} Q}{\otimes} A)(\Delta R) \neq\{0\}$. It follows from Corollary 6.4 that $\operatorname{Hom}_{\mathcal{F}}(R, Q)$ is non-empty. Exchanging the roles of $Q$ and $R$ yields an isomorphism $Q \cong R$ in $\mathcal{F}$. Suppose conversely that $Q \cong R$ in $\mathcal{F}$. By [22, 3.1], the existence of an isomorphism $Q \cong R$ in $\mathcal{F}$ is equivalent to the existence of an invertible element $a \in A^{\times}$and primitive idempotents $m \in A^{\Delta Q}, n \in A^{\Delta R}$ not contained in $\operatorname{ker}\left(\operatorname{Br}_{\Delta Q}\right)$, $\operatorname{ker}\left(\operatorname{Br}_{\Delta R}\right)$, respectively, such that ${ }^{a}(Q m)=R n$. Thus conjugation by $a$ induces an isomorphism $(m A m)(\Delta Q) \cong(n A n)(\Delta R)$, and since the blocks $e_{Q}, e_{R}$ of $k C_{G}(Q), k C_{G}(R)$, respectively, determined by $A$ are nilpotent, we have $k C_{P}(Q) \cong(m A m)(\Delta Q)$ and $k C_{P}(R) \cong(n A n)(\Delta R)$. Thus we get a commutative diagram of ring homomorphisms of the form

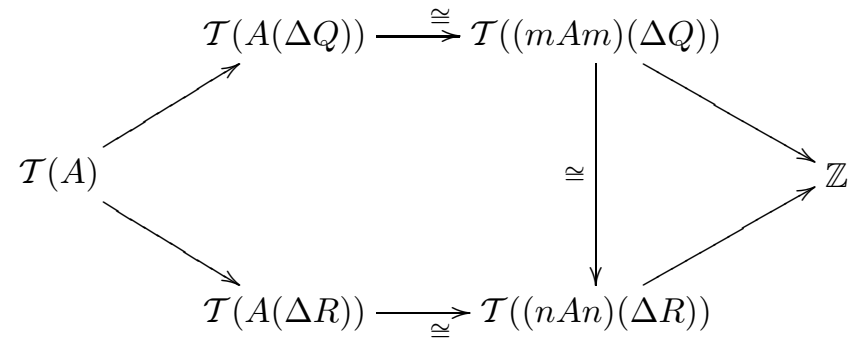

where the vertical arrow is induced by conjugation with $a$. The result follows.

\section{FurThER REMARKS}

13.1. One advantage of the concept of a $p$-permutation equivalence introduced by Boltje and $\mathrm{Xu} 4$ is that it admits the obvious source algebra formulation of Definition 1.3 above. The concept of isotypies, however, is formulated at the block algebra level, involving character values of group elements. Some of the technical issues in this paper are caused by the need to switch between block algebras and their source algebras; a version of isotypies in terms of source algebras would simplify some parts. The other advantage of $p$-permutation equivalences versus 
isotypies is that the rather technical compatibility conditions of an isotypy get replaced by a condition which is simpler in that it does not refer to the local structure of the blocks. It does not seem to be known whether every isotypy between two blocks can be "lifted" to a $p$-permutation equivalence.

13.2. Let $A, B$ be symmetric $\mathcal{O}$-algebras. Following [16, 2.9] any $A$-B-bimodule $M$ which is finitely generated projective as a left $A$-module and as a right $B$-module gives rise to a transfer map on Hochschild cohomology,

$$
t_{M}: H H^{*}(B) \longrightarrow H H^{*}(A) .
$$

This depends only on the isomorphism class of $M$. Thus, any element in $X \in$ $\mathcal{P}(A, B)$ determines a transfer map $t_{X}: H H^{*}(B) \longrightarrow H H^{*}(A)$. When applied to $A=B$ this yields a graded $\mathcal{P}(A)$-module structure on $H H^{*}(A)$ given by

$$
X \cdot \zeta=t_{X}(\zeta)
$$

for any $X \in \mathcal{P}(A)$ and any $\zeta \in H H^{*}(A)$. If $A$ is a source algebra of a block $b$ of some finite group with defect group $P$, then this module structure restricts to a $\mathcal{T}(A)$-module structure on $H H^{*}(A)$. By [16, 5.6.(iii)], the Hochschild cohomology algebra $H H^{*}(A)$ contains a canonical copy of the block cohomology $H^{*}(G, b)$. By [16, 6.7] this is a $\mathcal{T}(A)$-submodule of $H H^{*}(A)$.

13.3. Unlike the Burnside ring of a finite group, the trivial source bimodule ring of a block need not be commutative. Consider $p=2$ and $G=A_{4}=P \rtimes E$, where $P \cong C_{2} \times C_{2}$ and $|E|=3$. Then $\mathcal{O} G$ has a unique block. Write $\operatorname{Hom}\left(E, \mathcal{O}^{\times}\right)=$ $\left\{\mu_{0}, \mu_{1}, \mu_{2}\right\}$ with $\mu_{0}=1$. Every $\mu_{i}$ extends to an automorphism $\alpha_{i}$ of $\mathcal{O} G$ defined by $\alpha_{i}(e u)=\mu_{i}(e) u e$, for $u \in P$ and $e \in E$. These automorphisms fix $P$, and hence the three $\mathcal{O} G$-O $G$-bimodules $(\mathcal{O} G)_{\alpha_{i}}$ have vertex $\Delta P$ and trivial source. Thus they determine three elements in $\mathcal{T}(\mathcal{O} G)$. Setting $e_{i}=\frac{1}{3} \sum_{e \in E} \mu_{i}(e) e$ we get a primitive decomposition $\left\{e_{1}, e_{2}, e_{3}\right\}$ of 1 in $\mathcal{O} G$. One checks that $\alpha_{i}\left(e_{0}\right)=e_{i}$ and that $\alpha_{2}=$ $\left(\alpha_{1}\right)^{-1}$. Any algebra automorphism $\alpha$ of $\mathcal{O} G$ induces an isomorphism of bimodules ${ }_{\alpha} \mathcal{O} G \cong \mathcal{O} G_{\alpha-1}$ and isomorphisms of left $\mathcal{O} G$-modules ${ }_{\alpha} \mathcal{O} G j \cong \mathcal{O} G \alpha^{-1}(j)$ for any idempotent $j$ in $\mathcal{O} G$. Therefore

$$
\begin{aligned}
& {\left[(\mathcal{O} G)_{\alpha_{1}}\right]_{\dot{\mathcal{O}}_{G}}\left[O G e_{0} \underset{\mathcal{O}}{\otimes} e_{0} \mathcal{O} G\right]=\left[\mathcal{O} G e_{1} \underset{\mathcal{O}}{\otimes} e_{0} \mathcal{O} G\right],} \\
& {\left[O G e_{0} \underset{\mathcal{O}}{\otimes} e_{0} \mathcal{O} G \dot{\mathcal{O} G}_{\dot{\mathcal{O}}}\left[(\mathcal{O} G)_{\alpha_{1}}\right]=\left[O G e_{0} \underset{\mathcal{O}}{\otimes} e_{2} \mathcal{O} G\right] .\right.}
\end{aligned}
$$

In particular $\mathcal{T}(\mathcal{O} G)$ is not commutative.

\section{ApPendix: On stable Equivalences AND BlOCKS OF DEFECT 2}

The technology of this paper can be used to show that the proofs of two results due to R. Rouquier [27, 5.6, 6.3] for principal blocks carry over nearly verbatim to arbitrary blocks, giving in particular proofs of some of the results announced by Rouquier in [27, 6.4, Appendix]. For $A, B$ two symmetric $\mathcal{O}$-algebras, a bounded complex $X$ of $A$ - $B$-bimodules which are projective as left $A$-modules and as right $B$ modules is said to induce a stable equivalence if there are isomorphisms of complexes of bimodules $X \underset{B}{\otimes} X^{*} \cong A \oplus Y$ and $X^{*} \underset{A}{\otimes} X \cong B \oplus Z$ with $Y$ and $Z$ homotopy equivalent to bounded complexes of projective $A$ - $A$-bimodules and $B$ - $B$-bimodules, respectively. If $Y$ and $Z$ are homotopic to zero, then $X$ is called a Rickard complex. The following two theorems are [27, 5.6] and [27, 6.3] for arbitrary blocks. 
Theorem A.1. Let $G, H$ be finite groups, b, c blocks of $\mathcal{O} G, \mathcal{O} H$, respectively, having a common defect group $P$, and let $i \in(\mathcal{O} G b)^{\Delta P}$ and $j \in(\mathcal{O H} c)^{\Delta P}$ be source idempotents. Suppose that $i$ and $j$ determine the same fusion system $\mathcal{F}$ on $P$. For any subgroup $Q$ of $P$ denote by $e_{Q}$ and $f_{Q}$ the unique blocks of $k C_{G}(Q)$ and $k C_{H}(Q)$ satisfying $\operatorname{Br}_{\Delta Q}(i) e_{Q} \neq 0$ and $\operatorname{Br}_{\Delta Q}(j) f_{Q} \neq 0$. Let $X$ be a bounded complex of $\mathcal{O} G b-\mathcal{O} H$ c bimodules whose terms are finite direct sums of summands of the $\mathcal{O} G b-\mathcal{O H c}$-bimodules $\mathcal{O} G i \otimes j \mathcal{O} H$, where $Q$ runs over the subgroups of $P$. The following are equivalent:

(i) The complex $X$ induces a stable equivalence.

(ii) For every non-trivial subgroup $Q$ of $P$ the complex $e_{Q} X(\Delta Q) f_{Q}$ is a Rickard complex of $k C_{G}(Q) e_{Q}-k C_{H}(Q) f_{Q}$-bimodules.

Proof. Suppose that (i) holds. Write $X_{\mathcal{O} H c}^{\otimes} X^{*} \cong \mathcal{O} G b \oplus Y$ for some bounded complex $Y$ of $\mathcal{O} G b-\mathcal{O} G b$-bimodules which is homotopy equivalent to a complex of projective $\mathcal{O} G b-\mathcal{O} G b$-bimodules. Since the Brauer construction with respect to a non-trivial $p$-subgroup sends any projective module to zero and preserves homotopies, we get that $Y(\Delta Q) \simeq\{0\}$ for any non-trivial subgroup $Q$ of $P$. Thus, by [17, 2.3] (or by 9.2 translated back to block algebras via 4.7) we get that

$$
\begin{gathered}
\left(e_{Q} X(\Delta Q) f_{Q}\right) \underset{k C_{H}(Q) f_{Q}}{\otimes}\left(f_{Q} X^{*}(\Delta Q) e_{Q}\right) \cong e_{Q}\left(X_{\mathcal{O} H c}^{\otimes} X^{*}\right)(\Delta Q) e_{Q} \\
\simeq e_{Q}(\mathcal{O} G b)(\Delta Q) e_{Q}=k C_{G}(Q) e_{Q}
\end{gathered}
$$

for any non-trivial fully $\mathcal{F}$-centralised subgroup $Q$ of $P$. Since any subgroup of $P$ is isomorphic, in the fusion system $\mathcal{F}$, to a fully $\mathcal{F}$-centralised subgroup, we get this isomorphism for any non-trivial subgroup $Q$ of $P$. In particular, $e_{Q} X(\Delta Q) f_{Q}$ is a Rickard complex for any non-trivial subgroup $Q$ of $P$. Suppose conversely that (ii) holds. Let $Y$ be the mapping cone of the adjunction unit $\mathcal{O} G b \rightarrow X_{\mathcal{O} H c}^{\otimes} X^{*}$. By the assumptions, the induced map

$$
k C_{G}(Q) e_{Q} \rightarrow\left(e_{Q} X(\Delta Q) f_{Q}\right) \underset{k C_{H}(Q) f_{Q}}{\otimes}\left(f_{Q} X^{*}(\Delta Q) e_{Q}\right)
$$

is a homotopy equivalence, and so its mapping cone is contractible, for every nontrivial subgroup $Q$ of $P$. But as before, the right side is canonically isomorphic to $e_{Q}\left(X \underset{\mathcal{O} H c}{\otimes} X^{*}\right)(\Delta Q) e_{Q}$, and so the corresponding mapping cone is $e_{Q} Y(\Delta Q) f_{Q}$. This is contractible, for every non-trivial $Q$. But then in fact $Y(\Delta Q) \simeq 0$ for every non-trivial subgroup $Q$ of $P$, thanks to Proposition 4.8. A theorem of Bouc [5, 7.9] implies that $Y$ is homotopy equivalent to a bounded complex of projective bimodules, whence (i).

Theorem A.2. Let $G$ be a finite group, let $b$ be a block of $\mathcal{O} G$ with a defect group of order $p^{2}$, set $H=N_{G}(P)$ and denote by $c$ the block of $\mathcal{O} H$ satisfying $\operatorname{Br}_{\Delta P}(b)=$ $\operatorname{Br}_{\Delta P}(c)$. Let $i \in(\mathcal{O} G b)^{\Delta P}$ and $j \in(\mathcal{O H} c)^{\Delta P}$ be source idempotents such that $\operatorname{Br}_{\Delta P}(i)=\operatorname{Br}_{\Delta P}(j)$. There is a bounded complex of $\mathcal{O} G b-\mathcal{O} H c$-bimodules whose components are finite direct sums of summands of the bimodules $\mathcal{O} G i \otimes j \mathrm{O}$ O $Q$ running over the subgroups of $P$, such that $X$ induces a stable equivalence.

Proof. If $P$ is cyclic there is a Rickard complex of $\mathcal{O} G b-\mathcal{O} H c$-bimodules explicitly constructed by Rouquier [26]; it is observed in [17, $\$ 7$ ] that the components of this complex are as stated. Thus we may assume that $P$ is elementary abelian of rank 2 . 
The proof follows the lines of [27, 6.3]. Denote by $e_{Q}$ and $f_{Q}$ the unique blocks of $k C_{G}(Q)$ and $k C_{H}(Q)$, respectively, satisfying $\operatorname{Br}_{\Delta Q}(i) e_{Q} \neq 0$ and $\operatorname{Br}_{\Delta Q}(j) f_{Q} \neq 0$. Since $P$ is abelian, the fusion systems on $P$ determined by $i$ and by $j$ are equal to that of $k N\left(P, e_{P}\right) e_{P}$. Hence, for any subgroup $Q$ of $P$, we have

$$
N_{G}\left(Q, e_{Q}\right) / C_{G}(Q) \cong N_{H}\left(Q, f_{Q}\right) / C_{H}(Q)
$$

and both sides are $p^{\prime}$-groups. The blocks $e_{Q}, f_{Q}$ lift to unique blocks $\hat{e}_{Q}, \hat{f}_{Q}$ of $\mathcal{O} C_{G}(Q), \mathcal{O} C_{H}(Q)$, respectively. The images of $\hat{e}_{Q}, \hat{f}_{Q}$ in $\mathcal{O} C_{G}(Q) / Q, \mathcal{O} C_{H}(Q) / Q$ are blocks, denoted by $\bar{e}_{Q}, \bar{f}_{Q}$, respectively. If $Q$ has order $p$, then $\bar{e}_{Q}, \bar{f}_{Q}$ have the cyclic group $P / Q$ as a defect group, and $C_{H}(Q) / Q$ is the normaliser in $C_{G}(Q) / Q$ of $P / Q$. Thus $\bar{f}_{Q}$ is in fact the Brauer correspondent of $\bar{e}_{Q}$. By Rouquier's construction of Rickard complexes for blocks with cyclic defect groups in [26, 10.3], there is a Rickard complex of $\mathcal{O} C_{G}(Q) / Q \bar{e}_{Q}-\mathcal{O} C_{H}(Q) \bar{f}_{Q}$-bimodules $\bar{C}_{Q}$ of the form

$$
\bar{C}_{Q}=\cdots \rightarrow 0 \rightarrow \bar{N}_{Q} \stackrel{\bar{\Phi}_{Q}}{\longrightarrow} \bar{e}_{Q} \mathcal{O} C_{G}(Q) / Q \bar{f}_{Q} \rightarrow 0 \rightarrow \cdots
$$

for some projective bimodule $\bar{N}_{Q}$. This complex lifts to a Rickard complex of $\mathcal{O} C_{G}(Q) \hat{e}_{Q}-\mathcal{O} C_{H}(Q) \hat{f}_{Q}$-bimodules of the form

$$
C_{Q}=\cdots \rightarrow 0 \rightarrow N_{Q} \stackrel{\Phi_{Q}}{\longrightarrow} e_{Q} \mathcal{O} C_{G}(Q) f_{Q} \rightarrow 0 \rightarrow \cdots
$$

where $N_{Q}$ is a projective $\mathcal{O}\left(C_{G}(Q) \times \mathcal{O} C_{H}(Q)\right) / \Delta Q$-module lifting $\bar{N}_{Q}$, inflated to $\mathcal{O}\left(C_{G}(Q) \times C_{H}(Q)\right)$, and where $\Phi_{Q}$ lifts the map $\bar{\Phi}_{Q}$. By adapting arguments of Marcus [20,5.5] this complex extends to the group

$$
T=N_{G \times H}(\Delta Q) \cap\left(N_{G}\left(Q, e_{Q}\right) \times N_{H}\left(Q, f_{Q}\right)\right),
$$

and $T$ contains $C_{G}(Q) \times C_{H}(Q)$ as a normal subgroup of the $p^{\prime}$-index by the above remarks. Thus $N_{Q}$ remains projective when considered as an $\mathcal{O} T / \Delta Q$-module. We set

$$
V_{Q}=\operatorname{Ind}_{T}^{G \times H}\left(N_{Q}\right) .
$$

The inclusion $C_{G}(Q) \subseteq G$ induces an $\mathcal{O} T$-homomorphism $e_{Q} \mathcal{O} C_{G}(Q) f_{Q} \longrightarrow b \mathcal{O} G c$, which, by adjunction, yields a homomorphism of $\mathcal{O}(G \times H)$-modules

$$
\alpha_{Q}: \operatorname{Ind}_{T}^{G \times H}\left(e_{Q} \mathcal{O} C_{G}(Q) f_{Q}\right) \longrightarrow b \mathcal{O} G c .
$$

Set $\psi_{Q}=\alpha_{Q} \circ \operatorname{Ind}_{T}^{G \times H}\left(\Phi_{Q}\right): V_{Q} \rightarrow b \mathcal{O} G c$ and define the complex $X$ by

$$
X=\cdots \rightarrow 0 \rightarrow \underset{Q}{\oplus} V_{Q} \stackrel{\sum_{Q} \psi_{Q}}{\longrightarrow} b \mathcal{O} G c \rightarrow 0 \rightarrow \cdots
$$

with $b \mathcal{O} G c$ in degree zero, where $Q$ runs over a set of representatives of the $N_{G}\left(P, e_{P}\right)$-conjugacy classes of subgroups of order $p$ of $P$. If $Q, R$ are two subgroups of order $p$ which are not $N_{G}\left(P, e_{P}\right)$-conjugate, then $V_{R}(\Delta Q)=\{0\}$. This implies that $e_{Q} X(\Delta Q) f_{Q} \simeq C_{Q} \underset{\mathcal{O}}{\otimes}$, and this is a Rickard complex of $k C_{G}(Q) e_{Q^{-}}$ $C_{H}(Q) f_{Q^{-b i m o d u l e s . ~}}$ Moreover, $b \mathcal{O} G c$ is a direct summand of $\mathcal{O} G i \underset{\mathcal{O} P}{\otimes} j \mathcal{O H}$, and $V_{Q}$ is a direct sum of summands of $\mathcal{O} G i \underset{\mathcal{O} Q}{\otimes} j \mathcal{O H}$ because it is obtained from lifting, inflating and inducing a projective bimodule. Thus Theorem A.1 applies, showing that $X$ induces a stable equivalence.

\section{ACKNOWLEDGEMENT}

The author would like to thank the referee for his valuable comments. 


\section{REFERENCES}

1. J. L. Alperin, M. Broué, Local methods in block theory, Ann. of Math. 110 (1979), 143-157. MR.541333 (80f:20010)

2. R. Boltje, Chain complexes for Alperin's weight conjecture and Dade's ordinary conjecture in the abelian defect group case, J. Group Theory, to appear.

3. R. Boltje, B. Külshammer, A generalised Brauer construction and linear source modules, Trans. Amer. Math. Soc. 363 (2000), 3411-3428. MR.1694281 (2000j:20017)

4. R. Boltje, B. Xu, On p-permutation equivalences: between Rickard equivalences and isotypies, preprint (2006).

5. S. Bouc, Résolutions de foncteurs de Mackey, in: "Groups, representations: cohomology, group actions and topology" (eds.: A. Adem, J. Carlson, S. Priddy, P. Webb), Proc. Symp. Pure Math. 63 (1998), 31-83. MR.1603131 (99b:20015)

6. C. Broto, R. Levi, R. Oliver, The homotopy theory of fusion systems, J. Amer. Math. Soc. 16 (2003), 779-856. MR.1992826 (2004k:55016)

7. M. Broué, Brauer coefficients of p-subgroups associated with a p-block of a finite group, J. Algebra 56 (1979), 365-383. MR.528581 (80d:20013)

8. M. Broué, Isométries parfaites, types de blocs, catégories dérivées, Astérisque 181-182 (1990), 61-92. MR1051243 (91i:20006)

9. M. Broué, L. Puig, Characters and local structure in G-algebras, J. Algebra 63 (1980), 306317. MR $570714(81 \mathrm{j}: 20021)$

10. M. Broué, L. Puig, A Frobenius theorem for blocks, Invent. Math. 56 (1980), 117-128. MR558864 (81d:20011)

11. W. Feit, The representation theory of finite groups, North-Holland, Amsterdam, 1982. MR661045 (83g:20001)

12. R. Kessar, M. Linckelmann, On blocks of strongly p-solvable groups, Archiv Math., to appear. MR 2283678 (2007k:20023)

13. M. Linckelmann, The source algebras of blocks with a Klein four defect group, J. Algebra $\mathbf{1 6 7}$ (1994), 821-854. MR1287072 (95h:20014)

14. M. Linckelmann, On stable equivalences of Morita type, in: S. König, A. Zimmermann: "Derived Equivalences for Group Rings", Springer Lecture Notes in Mathematics 1685 (1998), 221-232. MR.1649847

15. M. Linckelmann, On derived equivalences and local structure of blocks of finite groups, Turkish J. Math. 22 (1998), 93-107. MR.1631770 (99k:20021)

16. M. Linckelmann, Transfer in Hochschild cohomology of blocks of finite groups, Algebras and Repr. Theory 2 (1999), 107-135. MR1702272 (2000h:20024)

17. M. Linckelmann, On splendid derived and stable equivalences of blocks of finite groups, J. Algebra 242 (2001), 819-843. MR 1848975 (2002i:20013)

18. M. Linckelmann, Simple fusion systems and the Solomon 2-local group, J. Algebra 296 (2006), 385-401. MR2201048 (2006i:20024)

19. M. Linckelmann, Introduction to fusion systems, in: Group Representation Theory (editors: M. Geck, D. Testerman, J. Thévenaz), EPFL Press, Lausanne (2007), 79-113. MR2336638

20. A. Marcuş, On equivalences between blocks of group algebras: reduction to simple components, J. Algebra 184 (1996), 372-396. MR1409219 (97m:20012)

21. L. Puig, Pointed groups and construction of characters, Math. Z. 176 (1981), 265-292. MR607966 (82d:20015)

22. L. Puig, Local fusion in block source algebras, J. Algebra 104 (1986), 358-365. MR866781 (88c:20020)

23. L. Puig, Nilpotent blocks and their source algebras, Invent. Math. 93 (1988), 77-116. MR.943924 (89e:20023)

24. L. Puig, On the local structure of Morita and Rickard equivalences between Brauer blocks, Progress in Mathematics 178 (Boston, Mass.), Basel: Birkhäuser, 1999. MR:1707300 (2001d:20006)

25. J. Rickard, Splendid equivalence: derived categories and permutation modules, Proc. London Math. Soc. 72 (1996), 331-358. MR.1367082 (97b:20011)

26. R. Rouquier, The derived category of blocks with cyclic defect groups, in: S. König, A. Zimmermann: "Derived Equivalences for Group Rings", Springer Lecture Notes in Mathematics 1685 (1998), 199-220. MR.1649846 
27. R. Rouquier, Block theory via stable and Rickard equivalences; in: Modular Representation Theory of Finite Groups, deGruyter, Berlin, 2001, pp. 101-146. MR.1889341 (2003g:20018)

28. J. Thévenaz, G-algebras and Modular Representation Theory, Oxford Science Publications, Clarendon, Oxford, 1995. MR1365077 (96j:20017)

Department of Mathematical Sciences, University of Aberdeen, Meston Building, Aberdeen, AB24 3UE, United Kingdom 